\title{
Antoine-Joseph Pernety, Cornelius de Pauw und die Berliner Debatte um die Neue Welt
}

Rollen wir die Berliner Debatte um die Neue Welt von ihren Anfängen im 18. Jahrhundert her auf, ohne gänzlich zu vergessen, dass der sogenannte 'Disput um die Neue Welt' bereits im Ausgang des 15. Jahrhunderts begann und bereits von Antonello Gerbi in einer klassischen Studie diskutiert wurde. ${ }^{1}$ Ich werde auf diese Studie noch kurz zurückkommen, will aber in einem ersten Schritt zunächst einmal die konkreten Ursprünge der neuen Debatte im 18. Jahrhundert suchen. Dabei ist es wichtig, dass wir uns mit der grundsätzlich neu erwachten und neu entfachten Aufmerksamkeit der europäischen Philosophen für die überseeische Problematik und vor allem für die weltweiten Beziehungen in dieser neuen, dieser zweiten Phase beschleunigter Globalisierung, vertraut machen.

Dabei möchte ich diese Debatte mit einer zunächst theoretischen Auseinandersetzung beginnen lassen, die einer der herausragenden Köpfe des 18. Jahrhunderts, der Genfer Bürger Jean-Jacques Rousseau, in Bewegung setzte. In einer der wohl wichtigsten Anmerkungen zu seinem Discours sur l'origine et les fondements de l'inégalité parmi les hommes setzte sich Rousseau im Jahre 1755 kritisch mit dem im Europa seiner Zeit erreichten Stand anthropologischer Kenntnisse auseinander.

Dabei hob er die unübersehbare, von den meisten der europäischen Philosophen aber sehr wohl übersehene Asymmetrie zwischen dem Wissensstand der aus den verschiedensten europäischen Ländern stammenden Reisenden einerseits und dem Reflexionsstand all jener Philosophen andererseits hervor, die ihre generalisierenden Überlegungen und Theorien zur Diversität des Menschengeschlechts nicht zuletzt auf der Grundlage mehr oder oftmals auch minder ausgedehnter und aufmerksamer Lektüren von Reiseberichten anstellten und entwickelten. Reiseberichte bilden oftmals die Grundlage für anthropologische sowie allgemein philosophische Betrachtungen, welche die conditio humana in einem weltweiten Maßstab im Jahrhundert der Aufklärung zu vergegenwärtigen suchten. Dass hierbei der Gegensatz zwischen den Reisenden und den Daheimgebliebenen eine fundamentale epistemologische Rolle spielte, ergibt sich im Rahmen dieser Vorlesung fast zwangsläufig. Doch gehen wir die Dinge systematisch an.

\footnotetext{
1 Vgl. Gerbi, Antonello: La disputa del nuovo mondo. Storia di una polemica: 1750-1900. Nuova edizione a cura di Sandro Gerbi. Con un profilo dell'autore di Piero Treves. Milano Napoli: Riccardo Ricciardi editore 1983.

¿ Open Access. () 2020 Ottmar Ette, publiziert von De Gruyter. (cc) BY-NC-ND Dieses Werk ist lizenziert unter der Creative Commons Attribution-NonCommericial-NoDerivatives 4.0 Lizenz.

https://doi.org/10.1515/9783110650686-018
} 


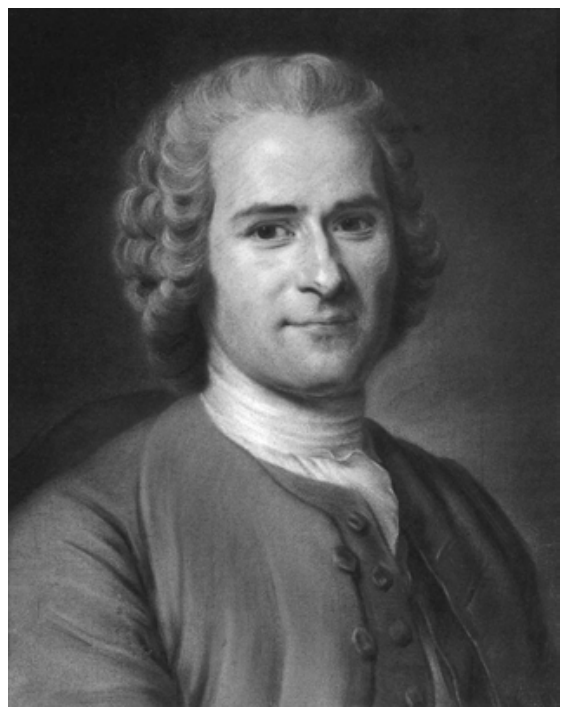

Abb. 65: Jean-Jacques Rousseau (Genf, 1712 - Ermenonville, 1778).

Jean-Jacques Rousseau betonte in diesem Zusammenhang die unabdingbare Notwendigkeit, diese fundamentale Asymmetrie innerhalb der weltweiten Zirkulation des Wissens zu durchbrechen, die im Übrigen nicht zuletzt durch die mangelnde Vorbereitung der allermeisten Reisenden verschärft werde. Er erkannte damit eines der epistemischen Fundamentalprobleme der Aufklärung, keine Frage. Zugleich aber hielt er auch einen gewissen Mangel hinsichtlich der (wissenschaftlichen) Ausrichtung mancher hommes éclairés fest, die sich dessen ungeachtet vielfältigen Gefahren ausgesetzt und lange, anstrengende Reisen unternommen hätten. Er bezieht sich dabei kritisch auf die Expeditionen der französischen Akademiemitglieder Mauperetuis und La Condamine, mit denen wir uns bereits beschäftigt haben:

Les Académiciens qui ont parcouru les parties Septentrionales de l'Europe et Méridionales de l'Amérique, avoient plus pour objet de les visiter en Géomètres qu'en Philosophes. Cependant, comme ils étoient à la fois l'un et l'autre, on ne peut pas regarder comme tout à fait inconnues les régions qui ont été vues et décrites par les La Condamine et les Maupertuis. ${ }^{2}$

2 Rousseau, Jean-Jacques: Discours sur l'origine et les fondements de l'inégalité parmi les hommes. In (ders.): Oeuvres complètes. Bd. III. Edition publiée sous la direction de Bernard Gagnebin et Marcel Raymond avec, pour ce volume, la collaboration de François Bouchardy, Jean-Daniel Candaux, Robert Derathé, Jean Fabre, Jean Starobinski et Sven Stelling-Michaud. Paris: Gallimard 1975, S. 213. 
Der Verfasser des Discours sur l'inégalité, wie dieser Second Discours auch in abgekürzter Form oft benannt wird, ließ keinerlei Zweifel aufkommen: Nicht von den Geometern, sondern von den Philosophen erhoffte sich Rousseau die entscheidenden Verbesserungen des Kenntnisstandes über die außereuropäische Welt. Denn dieser Kenntnisstand, dies wusste der Genfer Philosoph, war in Europa noch immer erschreckend niedrig. Räumte Rousseau auch gerne Gehalt und Qualität mancher Reiseberichte ein, die im 18. Jahrhundert veröffentlicht worden waren, so verbarg er doch seine fundamentale Kritik am allgemeinen Niveau anthropologischer beziehungsweise ethnologischer Kenntnisse nicht.

Dabei nahm er bei aller Bewunderung für das Werk eines Buffon auch den Bereich der Naturgeschichte und insbesondere der von ihren Vertretern genutzten Quellen nicht aus von seiner Kritik. Nach der Erwähnung einiger weniger glaubwürdiger Berichte stellte er mit aller wünschenswerten Deutlichkeit fest:

A ces relations près, nous ne connoissons point les Peuples des Indes Orientales, fréquentées uniquement par des Européens plus curieux de remplir leurs bourses que leurs têtes. [...] toute la terre est couverte de Nations dont nous ne connoissons que les noms, et nous nous mêlons de juger le genre humain! Supposons un Montesquieu, un Buffon, un Diderot, un Duclos, un d'Alembert, un Condillac, ou des hommes de cette trempe voyageant pour instruire leurs compatriotes, observant et décrivant comme ils savent faire, la Turquie, l'Egipte, la Barbarie, l'Empire de Maroc, la Guinée, les pays des Caffres, l'intérieur de l'Afrique et ses côtes Orientales [...]: puis dans l'autre Hémisphére le Méxique, le Perou, le Chili, les Terres Magellaniques, sans oublier les Patagons vrais ou faux [...]; supposons que ces nouveaux Hercules, de retour de ces courses mémorables, fissent ensuite à loisir l'Histoire naturelle, Morale et Politique de ce qu'ils auroient $v u$, nous verrions nous mêmes sortir un monde nouveau de dessous leur plume, et nous apprendrions ainsi à connoître le nôtre. ${ }^{3}$

Hier macht Rousseau gleichsam eine Wunschliste auf mit all jenen Gebieten, über die man unbedingt mehr wissen müsse, um sich ein Bild von der Welt und zugleich auch ein besseres Bild vom Eigenen machen zu können. Aufgrund ihrer alles beherrschenden persönlichen wie kommerziellen Interessen entgehen die meisten europäischen Reisenden mit ihren so zahlreichen, aber oft auch so ungesicherten und vor allem interessegeleiteten Berichten dem letztlich vernichtenden Urteil Rousseaus nicht. Dies sind in der Tat Reiseberichte, die wir in dieser Vorlesung größtenteil links liegen ließen, da sie nur der Erfüllung spezifischer Interressen von Kaufleuten oder Mönchen, Soldaten oder Abenteurern entsprangen.

Doch der Verfasser des Diskurses über die Ungleichheit leugnete die grundlegende Bedeutung der Reisen für die Ausweitung der menschlichen Kenntnisse und die umfassende Zirkulation von Wissen im europäisch-außereuropäischen

3 Ebda., S. 213. 
Spannungsfeld keineswegs. Ganz im Gegenteil: Für ihn ging es darum, wohlvorbereitete Reisende, 'Philosophen' (im Sinne des 18. Jahrhunderts) auszusenden, welche über einen wesentlich höheren Grad an Glaubwürdigkeit verfügen sollten. Sie sollten nicht nur wissenschaftlich vorgehen, sondern auch philosophisch denken und versuchen, das von ihnen bezüglich der bereisten Gebiete gesammelte Wissen in eine generelle Weltanschauung zu integrieren. Sie sollten überdies nicht nur über ein Wissen (savoir), sondern mehr noch über ein savoir faire und ein savoir voir verfügen und in der Lage sein, nach ihrer Rückkehr nach Europa das, was sie gesehen hatten, ebenso an ihre Landsleute wie an ihr Lesepublikum innerhalb einer tendenziell weltumspannenden République des Lettres der Aufklärung weiterzugeben. Denn nur auf diese Weise sei es möglich, neue Grundlagen für ein neues Wissen zu schaffen. Rousseau verlangte also in der Tat viel von den 'neuen Reisenden'. Aber war dies nicht zuviel verlangt?

Halten wir fest: Die Vorstellungen Rousseaus waren von größter epistemologischer Tragweite. Savoir faire und savoir voir sollten auf diese Weise für die künftigen Leser des Reisenden in ein savoir faire voir, ein Wissen und eine Technik des Vor-Augen-Führens, umschlagen, das nicht nur die europäische Sichtweise der Neuen Welt modifizieren und den Nouveau-Monde in einen monde nouveau verwandeln, sondern den Blick auch auf die europäischen Länder selbst grundlegend verändern könnte. Rousseau hatte verstanden, dass ein veränderter Blick auf die außereuropäische Welt auch einen veränderten Blick auf Europa beinhalten musste. Man könnte hier auch von einer Dialektik und wechselseitigen Abhängigkeit von Fremd- und Selbstwahrnehmung sprechen.

Um es allgemeiner zu sagen: Fremderkenntnis impliziert stets Selbsterkenntnis; und mehr noch: Selbsterkenntnis ist ohne Fremderkenntnis nicht zu haben. Beide stehen zueinander in Wechselwirkung. Im Übrigen kann nicht übersehen werden, dass innerhalb einer weltweiten - wenn auch zweifellos von Europa beherrschten - Relationalität die Kenntnisse reisender europäischer Philosophen auch für die Bewohner anderer Areas und Weltregionen notwendig von Nutzen sein mussten. Rousseaus Argumente sind jener Ethik mit universalisierendem Anspruch verpflichtet, wie sie der (europäischen) République des Lettres zugrunde lag. Dieser Aspekt ist sicherlich Teil eines lebendigen Erbes der Aufklärung und im Habermas'schen Sinne genuiner Bestandteil eines unvollendeten Projekts der anhebenden Moderne. Denn dass wir in dieser Vorlesung das letzte Viertel des 18. Jahrhunderts als den Beginn eines Prozesses sehen, den wir als die europäische Moderne bezeichnen, sollte - auch wenn wir in dieser Vorlesung nicht speziell auf die unterschiedlichen Definitionen eingehen können, um die Moderne zu fassen - insgesamt klar und verständlich sein. Aber ich werde darauf in der gebotenen Kürze zurückkommen. 
Zurück zum roten Faden unserer Vorlesung! In der angeführten Passage erscheinen Sehen und Schreiben in ihrer Verbindung mit dem Reisen als komplementäre Handlungen, die in ihrer Abfolge einen Sinn (bezüglich) der Neuen Welt hervorbringen, den Rousseau dann auch zu glauben bereit wäre: „il faudra les en croire“. ${ }^{4}$ Doch gründet dieser Glaube für Rousseau nicht auf dem Schreiben, der écriture, allein. Nicht die Bewegung des Diskurses, sondern die Bewegung des Reisens, das eine direkte Sicht auf die Dinge gewährt, jene Ortsveränderung also, die ein unmittelbares Sehen des Anderen ermöglicht, verleiht dem Schreiben über das Andere Autorität und damit erst eine Autorschaft im starken Sinne. Reisen, das Wissen aus der Bewegung, erscheint hier in seiner ganzen Stärke bei Rousseau. Die Glaubwürdigkeit dieser Autorschaft ist in diesen Passagen des Second Discours folglich an die Legitimation durch eine Augenzeugenschaft zurückgebunden, die mit Blick auf das Wissen über weit entfernte Länder das eigene Reisen zwingend voraussetzt. Wir stoßen hier auf eine zentrale Achse des Verhältnisses zwischen Reisen und Schreiben, das gerade auch für diesen dritten Teil unserer Vorlesung von enormer Bedeutung ist.

Ein Gedanke glimmt hier auf, der für unsere Zeit, für unsere eigenen wissenschaftlichen und wissenschaftspolitischen Epistemologien (und 'Selbstverständlichkeiten') von größter Aktualität und Dringlichkeit ist. Denn über lange Zeit - und bis in unsere Gegenwart - hielt sich die Vorstellung, man habe die 'allgemeine', die generalisierende Theorie von den sogenannten Regionalwissenschaften, den Area Studies, als 'systematische' oder systematisierende Wissenschaft klar zu trennen, da letztere nur ein regional begrenztes Wissen - etwa über die Türkei, Nordafrika oder Südamerika, um bei den Beispielen Rousseaus zu bleiben - hervorzubringen in der Lage wären. Wie aber wäre ein solcher Glaube zu begründen? Oder noch stärker: Welche Gründe ließen sich für einen derartigen akademischen Irrglauben auftreiben?

Denn nichts ist - und auch dies wird die Berliner Debatte um die Neue Welt in der historischen Rückschau zeigen - anmaßender und den Gegenständen inadäquater als eine derartige künstliche Trennung. Die vorgeblich 'allgemeine' Theorie beruht zumeist auf äußerst lückenhaften Kenntnissen, die sich in der Regel auf das Wissen über einen mehr oder minder kleinräumigen und bestenfalls europäischen Ausschnitt beschränken, der ungerührt und unhinterfragt als normgebend gesetzt wird.

4 Rousseau, Jean-Jacques: Discours sur l'origine et les fondements de l'inégalité parmi les hommes, S. 214. 
Eine solche Setzung aber ist durch keine Begründung zu legitimieren. Sie beruht allein auf akademischen Gepflogenheiten, auf der selbstverständlichen, aber nicht mehr länger ausgesprochenen Annahme, dass die Werte Europas universell gültig seien. Um aber eine allgemeine Theorie adäquat fundieren und entwickeln zu können, ist die Kenntnis verschiedenster Areas, verschiedenster Weltregionen, unverzichtbar. Denn dann erst kann die Zielvorstellung Rousseaus greifen, unsere Welt in ihrer Gesamtheit auf neue Weise zu erfassen: „nous verrions nous mêmes sortir un monde nouveau de dessous leur plume, et nous apprendrions ainsi à connoître le nôtre“. 5 Jean-Jacques Rousseau hätte es nicht besser formulieren können, was die Rückwirkungen auf Europa zwingend sein mussten.

Man könnte den ausführlich zitierten Überlegungen Rousseaus eine Passage aus der Feder des von ihm im obigen Zitat erwähnten Diderot an die Seite stellen, die erstmals ${ }^{6} 1780$ im elften Buch der dritten Ausgabe der Histoire philosophique et politique des établissements et du commerce des européens dans les deux Indes veröffentlicht wurde. In gewissem Sinne handelt es sich um eine Antwort und mehr noch um eine scharfsinnige erkenntnistheoretische Replik auf den zweiten Discours durch einen Rousseau kritisch gegenüberstehenden französischen Philosophen fünfundzwanzig Jahre nach dessen Veröffentlichung.

Denn zu Beginn von Rousseaus Überlegungen findet sich seine Klage darüber, niemals „deux hommes bien unis, riches, l'un en argent, l'autre en génie, tous deux aimant la gloire“ gefunden $\mathrm{zu}$ haben, einen Ruhm, für den der eine bereit wäre, 'zwanzigtausend Taler' zu opfern, der andere 'zehn Jahre seines Lebens' zugunsten einer erfolgreichen Reise um die Welt. ${ }^{7}$

Uns interessiert hier nicht die bemerkenswerte Beziehung zwischen den Zahlenangaben, sondern die Tatsache, dass Diderot die diskursive Struktur dieser Passage wiederaufnahm, um zugleich ihrem kritischen Sinn eine neue Wendung zu geben:

L'homme riche dort; le savant veille; mais il est pauvre. Ses découvertes sont trop indifférentes aux gouvernemens pour qu'il puisse solliciter des secours ou espérer des récompenses. On trouveroit parmi nous plus d'un Aristote; mais où est le monarque qui lui dira: ma puissance est à tes ordres $[\ldots] .^{8}$

5 Ebda., S. 213.

6 Vgl. Duchet, Michèle: Diderot et l'Histoire des Deux Indes ou l'Ecriture Fragmentaire. Paris: Nizet 1978, S. 84.

7 Ebda., S. 213.

8 Raynal, Guillaume-Thomas: Histoire philosophique et politique des établissements et du commerce des européens dans les deux Indes. Tome cinquième. Genève: Chez Jean-Léonard Pellet, Imprimeur de la Ville \& de l'Académie 1781, S. 43. 
Ein Vierteljahrhundert nach dem Erscheinen des zweiten Discours Rousseaus ist die Verbindung zwischen dem Reichen und dem Genie, zwischen dem Monarchen und dem Gelehrten, schlicht zwischen Reisen und Schreiben nicht realistischer geworden. Zwischen beiden Polen von Reisen und Schreiben klafft eine epistemologische Lücke, welche die so wichtige und transformierende Wechselwirkung zwischen beiden Elementen verhindert.

Anders als Rousseau, der am liebsten einen Buffon, aber auch einen Diderot auf eine Weltreise geschickt hätte, war der Ko-Autor von Guillaume-Thomas Raynals Histoire des deux Indes in keiner Weise dazu bereit, sein Arbeitszimmer in Paris zu verlassen und die Welt zu umsegeln. Und in einer Passage aus seiner Feder führte er hierzu keineswegs persönliche, sondern epistemologische Gründe an:

L'homme contemplatif est sédentaire; \& le voyageur est ignorant ou menteur. Celui qui a reçu le génie en partage, dédaigne les détails minucieux de l'expérience; \& le faiseur d'expériences est presque toujours sans génie. ${ }^{9}$

Bei Jean-Jacques Rousseau basiert das Wissen auf dem Sehen, das sa-voir auf dem voir. Ist die Verbindung zwischen dem mit Geld und dem mit Genie gesegneten Menschen zufälliger Natur, so ist die Einheit zwischen dem Philosophen und dem Reisenden, zwischen philosophe und voyageur für Rousseau bewusst und konzeptionell fundiert: Sie ist Programm.

Wir finden bei Diderot die Spaltung zwischen dem Mächtigen und dem Gelehrten zwar wieder, doch unternimmt dieser savant keine physischen Reisen, sind seine Bewegungen doch rein geistiger Natur und bündeln sich zwischen den vier Wänden seines Arbeitszimmers. Denis Diderot weist ihm einen Ort zu, den er nicht verlassen wird: Der Ort des Arbeitens und der Ort des Schreibens unterscheiden sich in einem rein räumlichen Sinne nicht voneinander. Reisen und Schreiben aber sind weit auseinander gerückt.

Dem homo contemplativus stellt Diderot nicht den homo faber, sondern den Reisenden, eine Art homo migrans, gegenüber, der entweder unter einem Mangel an Wissen (ignorant) oder unter einem Mangel an Wahrheit und Wahrhaftigkeit (menteur) leide und daher für Diderot in einem System hierarchisierter Wissenszirkulation auf eine bestenfalls zweitrangige Bedeutung herabgestuft werden muss. Das Reisen ist für Diderot sekundär, absolut zweitrangig. A beau mentir qui vient de loin - und man könnte aus heutiger Sicht fast glauben,

9 Raynal, Guillaume-Thomas: Histoire philosophique et politique des établissements et du commerce des européens dans les deux Indes. Tome cinquième. Genève: Chez Jean-Léonard Pellet, Imprimeur de la Ville \& de l'Académie 1781, S. 43. 
dieses Sprichwort sei auch geprägt worden für jene Wissenschaften, die vor wenigen Jahren einmal ein wenig unglücklich als 'Fernwissenschaften' bezeichnet wurden. Glücklicherweise konnte sich dieser Begriff nicht halten: $\mathrm{Zu}$ deutlich ist in ihm die strikt eurozentrische Sichtweise verankert, die das 'Ferne' nur aus dem Abstand zu Europa bemisst.

Doch bleiben wir im 18. Jahrhundert und sehen wir uns die dortigen Herausforderungen an. Nicht umsonst ließ Denis Diderot die Dialogpartner seines Supplément au voyage de Bougainville darüber debattieren, ob der berühmte französische Entdeckungsreisende bei seiner Weltumsegelung nicht eher ein sesshafter Bewohner auf den Planken seines schwimmenden Hauses („maison flottante“10) gewesen sei, während der Leser von Bougainvilles berühmtem Reisebericht als der eigentliche Weltreisende betrachtet werden müsse, sei er es doch, der - scheinbar unbeweglich auf den Dielen seines fest gebauten Hauses - kraft seiner Lektüre die Welt umrundet habe. ${ }^{11}$ Man könnte hier mit guten Gründen gewiss nicht von einem Diderot'schen paradoxe sur le comédien, wohl aber vom Paradox über den Reisenden, vom paradoxe sur le voyageur sprechen, welcher in seiner höchsten Form der Leser ist - und wäre er ein Leser des Buches der Welt. ${ }^{12}$ Diderot stellt auf eine kluge und zugleich recht durchtriebene Weise das Verhältnis zwischen Reisen und Schreiben in seinem Paradoxe sur le voyageur auf den Kopf.

Ohne an dieser Stelle die Tatsache ausführen zu können, dass die epistemologisch so relevante Scheidung zwischen den Reisenden und den Daheimgebliebenen keineswegs eine Erfindung des 18. Jahrhunderts war, sondern sich wie wir sahen - seit der ersten Phase beschleunigter Globalisierung durch den gesamten abendländischen Diskurs über die Neue Welt zog und im Übrigen bereits in der Antike hinsichtlich des Zusammenspiels von Auge und Ohr als Quellen der Information über eine unbekannte Welt präsent war, ${ }^{13}$ sei doch darauf verwiesen, dass jenseits des hier markierten und in der Tat markanten epistemologischen Gegensatzes zwischen Rousseau und Diderot die beiden großen Philosophen der europäischen Aufklärung in einem nicht unwesentlichen

10 Diderot, Denis: Supplément au Voyage de Bougainville ou Dialogue entre A et B. In (ders.): Oeuvres. Edition établie et annotée par André Billy. Paris: Gallimard 1951, S. 964.

11 Vgl. hierzu Ette, Ottmar: 'Le tour de l'univers sur notre parquet': lecteurs et lectures dans l’Histoire des deux Indes'. In: Bancarel, Gilles / Goggi, Gianluigi (Hg.): Raynal, de la polémique à l'histoire. Oxford: Voltaire Foundation 2000, S. 255-272.

12 Vgl. Blumenberg, Hans: Die Lesbarkeit der Welt. Frankfurt am Main: Suhrkamp 1986.

13 Vgl. zu diesen Zusammenhängen umfassender das dritte Kapitel in Ette, Ottmar: Literatur in Bewegung. Raum und Dynamik grenzüberschreitenden Schreibens in Europa und Amerika. Weilerswist: Velbrück Wissenschaft 2001. 
Punkt miteinander übereinstimmen. Denn der reisende Philosoph, der philosophe voyageur Rousseaus wählt ganz wie der sesshafte homo contemplativus Diderots denselben Ort, um sein Werk niederzuschreiben: den europäischen Schreibtisch, sein Arbeitszimmer in Europa.

Das hermeneutische Bewegungsmuster der Reise und deren genuines Verhältnis zum Schreiben beruht für beide europäische philosophes als Verstehensprozess stets auf einem Kreis, auf einem hermeneutischen Zirkel. Folglich ist es auch keineswegs zufällig, dass Rousseau in der oben angeführten Passage von einer Reise um die Welt spricht, gleichsam dem Muster der europäischen Entdeckungsreise ab der Mitte des 18. Jahrhunderts. Ist der reisende Philosoph erst einmal nach Hause zurückgekehrt und aufgrund seiner Mühen und seines Leidens in einen 'neuen Herkules' verwandelt, wird er sich bald an seinen Schreibtisch setzen und seine Ansichten niederschreiben. Reisen und Schreiben stehen hier in einem klar definierten Verhältnis zueinander, das durch Nachzeitigkeit und gerade nicht durch Simultaneität geprägt ist. Erst kommt das Reisen (durch die Welt), dann folgt das Schreiben (notwendig in Europa).

Dass die Niederschrift des Textes, die eigentliche Textproduktion, 'selbstverständlich nicht in Übersee, sondern in Europa erfolgt, erscheint beiden Europäern als geradezu natürlich. Es ist die zweite Natur der europäischen Philosophie. Denn der Ort des Schreibens kann als Ort der Wissensproduktion innerhalb eines zutiefst asymmetrischen Zirkulationssystems von Wissen nur in Europa und letztlich nur in einer der europäischen Metropolen des Wissens angesiedelt sein. Das Lesen im Buch der Natur mag oder muss weltweit erfolgen; die Niederschrift dieser Lektüre der Natur aber kann - dies braucht weder ein Rousseau noch ein Diderot zu thematisieren - 'natürlich' nur in den europäischen Zentren des Wissens erfolgen. Die ganze Welt mag ein Reich der Zeichen sein: Europa aber versteht sich als das Reich des Wissens, das diese Zeichen zu interpretieren weiß.

Innerhalb der hier von Jean-Jacques Rousseau und Denis Diderot skizzierten Problematik der Beziehung zwischen Reisen und Wissen sowie Reisen und Schreiben situiert sich auch die Berliner Debatte um die Neue Welt, die als ein eminent wichtiger Teil des von dem bereits erwähnten Antonello Gerbi in einer längst kanonisch gewordenen Studie so bezeichneten 'Disputs um die Neue Welt ${ }^{, 14}$ angesehen werden muss. Aber sie bildet eine neue, eigene Phase, die ganz zweifellos mit der zweiten Phase beschleunigter Globalisierung verbunden und eng verknüpft ist. Ohne diese zeitgenössisch beschleunigte Phase wäre sie

14 Vgl. Gerbi, Antonello: La disputa del nuovo mondo. Storia di una polemica: 1750-1900 (1983). 
undenkbar: Denn sie bietet unterschiedliche Antworten auf die Herausforderungen dieser neuen Phase.

Aber kommen wir zunächst einmal zu einem der Protagonisten dieser Berliner Debatte. Er ist, wie auch sein Gegenspieler, kein Berliner. Was nicht verwundert, war Berlin zum damaligen Zeitpunkt doch nicht gerade ein Zentrum weltweiter Globalisierungseffekte, sondern eher eine aufstrebende mittelgroße Stadt inmitten einer Sandwüste und beseelt von dem Aufstiegswillen ihres ambitionierten Königs Friedrich dem Zweiten oder, wenn Sie so wollen, dem Großen. Ja, und dieses eher beschauliche Berlin nun wurde zum Schauplatz einer Debatte mit weltumspannenden Konsequenzen.

Einer der beiden wichtigsten Protagonisten dieser nicht nur in Europa, sondern auch in Übersee mit größter Aufmerksamkeit registrierten und kommentierten Berliner Debatte, Antoine-Joseph Pernety, war einst in der Funktion eines Schiffskaplans mit keinem Geringeren als Bougainville zwar nicht um die gesamte Erdkugel, wohl aber in die einst von Amerigo Vespucci so genannte 'Neue Welt' gereist. Diese Tatsache sollte in jenen Auseinandersetzungen, die in der aufstrebenden Hauptstadt des ehrgeizigen Preußenkönigs - der mit seinem Libretto für die am 6. Januar 1755 erstmals in der Lindenoper aufgeführten Oper Montezuma selbst eine Art sinnlich-machtpolitisches 'Vorspiel' für diese Debatte lieferte ${ }^{15}$ ihren Ausgang nahmen, aber rasch auf ein weltweites Echo stießen, eine wichtige Rolle spielen.

Aber worum ging es in dieser Debatte? Mit einigen der zentralen Fragen aufklärerischer Anthropologie, aber auch mit den hier dargestellten Problemen wahrnehmungstheoretischer Epistemologie beschäftigte sich der früher von der Inquisition in Avignon verfolgte und nach Preußen geflüchtete Benediktiner Antoine-Joseph Pernety bereits in seinem 1769 in französischer Sprache zu Berlin erschienenen Journal historique. Es stieß auf keine besonders große Resonanz in der aufklärerischen République des Lettres. Darin berichtete der auf der Titelseite stolz als „Membre de l'Académie Royale des Sciences \& Belles-Lettres de Prusse“ und als „Bibliothécaire de Sa Majesté le Roy de Prusse“ Bezeichnete von seiner Reise unter der Leitung von Louis-Antoine de Bougainville zu den tropischen Küsten des heutigen Brasilien, zu den Malwinen-Inseln und an die Südspitze des amerikanischen Kontinents. ${ }^{16}$ Pernety hatte also eine veritable Weltreise hinter sich gebracht.

15 Vgl. hierzu das vierte Kapitel in Ette, Ottmar: Mobile Preußen. Ansichten jenseits des Nationalen. Stuttgart: Metzler 2019.

16 Vgl. Pernety, Antoine-Joseph: Journal historique d'un Voyage aux Iles Maloü̈nes en 1763 \& 1764, pour les reconnoître, \& y former un établissement; et de deux Voyages au Détroit de Magellan, avec une Relation sur les Patagons. 2 Bde. Berlin: Etienne de Bourdeaux 1769. 
In seinem Journal betonte Pernety immer wieder die grundsätzliche Andersartigkeit der Neuen Welt. Damit stand er durchaus im Einklang mit seinen Zeitgenossen, für welche der Gegensatz zwischen beiden Hemisphären ebenfalls ein ganz grundsätzlicher war. So schilderte er auch auf nicht weniger als zwanzig Seiten die am 10. November 1763 erfolgte Querung des Äquators und damit jene Szenerie, die sich so oder in vergleichbarer Form auf allen französischen wie europäischen Schiffen vollzog, welche die Äquatoriallinie, den Zentralbereich des Tropengürtels, passierten und in eine andere Hemisphäre eintraten. Dieser Übergang markierte den Wechsel von der einen Welt in eine gänzlich andere.

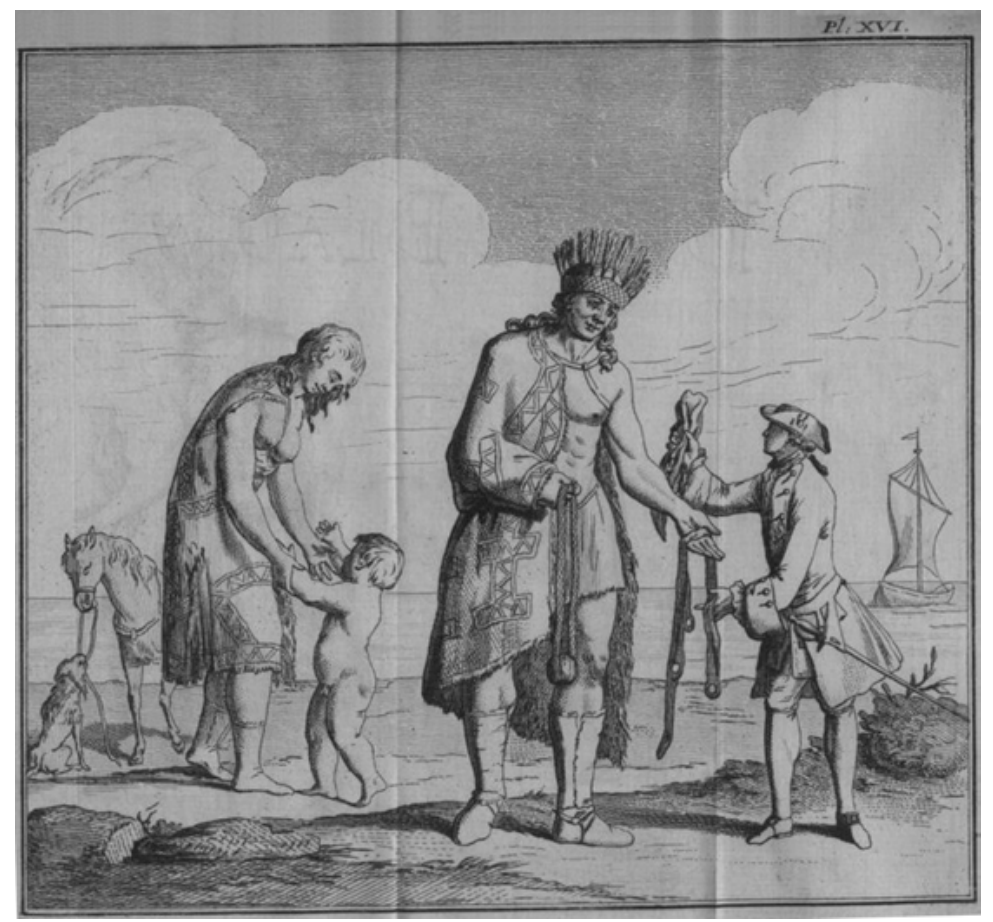

Abb. 66: Antoine-Joseph Pernety (Roanne, 1716 - Avignon, 1796) und die Patagonen in Pernetys Journal historique.

Längst waren die Tropen für die europäischen Seefahrtsnationen zum planetarischen Bewegungs-Raum par excellence geworden, ${ }^{17}$ doch stellten sie damit

17 Vgl. hierzu Ette, Ottmar: Diskurse der Tropen - Tropen der Diskurse: Transarealer Raum und literarische Bewegungen zwischen den Wendekreisen. In: Hallet, Wolfgang / Neumann, 
zugleich den Schwellenbereich eines Übergangs dar, den man bei diesem sich auf Südkurs nach Brasilien befindenden Schiff als einen symbolträchtigen Übergang von der östlichen in die westliche Hemisphäre, gleichzeitig aber auch von der nördlichen auf die südliche Halbkugel beschreiben darf. Diesen doppelten Übergang markiert der Reisebericht des Franzosen auf durchaus eindrucksvolle Weise. Beschäftigen wir uns daher kurz mit diesem Abschnitt seines durchaus spannenden Textes.

Pernetys ausführliche Darstellung des „Baptême de la ligne“, ${ }^{18}$ das noch in der glühenden Mittagshitze an Deck des französischen Schiffes mit der Aufstellung eines Beckens mit Meerwasser, eines Throns für den „Seigneur Président de la Ligne“ sowie weiterer Sitzgelegenheiten für den „Chancelier“ sowie den (aus Sicht des aumônier sicherlich besonders interessanten) „Vicaire“ nebst einer Versammlung der gesamten Mannschaft und aller Passagiere begann, ${ }^{19}$ hebt detailreich und nicht ohne literarisches Geschick den zeremoniellen und rituellen Charakter dieser Äquatortaufe hervor. Die karnevalesken Züge der Szenerie sind unübersehbar, betritt doch zunächst - selbstverständlich mit dem Einverständnis Kapitän Bougainvilles - ein von sechs Schiffsjungen sekundierter und in einen Schafspelz gehüllter und bemalter Matrose, dessen Verkleidung und Verwandlung präzise beschrieben wird, ${ }^{20}$ die Bühne, bevor der ebenfalls in Schafsfelle gekleidete bon-homme de la Ligne höchstselbst, umgeben von seinem herausgeputzten Hofstaat, das Kommando an Bord übernimmt. Das Schauspiel travestierter Alterität beginnt. Karnevalesk ist es auch in dem Sinne, dass es uns einen monde à l'envers, eine Welt auf dem Kopf, zeigt.

Die Querung der Äquatoriallinie stellt vergleichbar mit dem Zeit-Raum des Karnevals die 'natürlichen' Hierarchien also gleichsam auf den Kopf: Eine Tatsache, die das karnevaleske Element mit der Vorstellung der Antipoden - einer Welt, deren Bewohner buchstäblich auf dem Kopf stehen und in der die Bäume nach unten wachsen - zu verbinden scheint. Dem Lesepublikum wird auf diese Weise anschaulich vor Augen geführt, dass es dem Reisenden in eine andere, in eine fremde Welt folgt, die über einen eigenen Zeichenvorrat verfügt. Nichts scheint in dieser 'anderen' Welt gleich zu sein, alles muss sich hier notwendig unterscheiden. Es lohnt daher, der literarischen Ausgestaltung dieser Szenerie, die zweifellos im Sinne unserer Vorlesung den reiseliterarischen Ort eines

Birgit (Hg.): Raum und Bewegung in der Literatur. Die Literaturwissenschaften und der Spatial Turn. Bielefeld: transcript Verlag 2009, S. 139-165.

18 Pernety, Antoine-Joseph: Journal historique d'un Voyage aux Iles Malouïnes en 1763 \& 1764, Bd. 1, S. 95.

19 Ebda., S. 96.

20 Ebda., S. 98 f. 
Höhepunkts von Pernetys Journal historique markiert, etwas Aufmerksamkeit zu schenken.

Ohne die von Pernety entworfene literarische Szene in all ihren Details wiedergeben zu können, ist es doch aufschlussreich zu beobachten, dass die Inszenierung einer Gegen-Ordnung, die für einen Tag zwischen den Wendekreisen symbolisch in Kraft tritt, jene Wendungen und Wandlungen vorführt, welche die Tropen als gegenüber Europa andere Welt im Zeichen der Trennlinie des Äquators repräsentieren. Die Tropen sind in diesem Sinne auch rhetorisch eine andere Figur.

Inwieweit diese andere Ordnung aus einer ihrerseits nicht unterlaufenen patriarchalischen Perspektive zugleich auf das 'andere' Geschlecht projiziert wird, mag das folgende Zitat der fruchtbaren 'Versprechen' des Kapitäns belegen:

Les choses ainsi disposées, le Vicaire dit à Mr. de Bougainville: 'Pour être reçu dans la noble \& puissante société du Seigneur Président de la Ligne, il faut prendre, au préalable, quelques engagemens, que vous promettez d'observer. Ces engagemens n'ont pour objet que des choses raisonnables. A la bonne heure, répondit Mr. de Bougainville. Promettezvous, dit alors le Vicaire, d'être bon Citoyen, \& pour cet effet de travailler à la population, \& de ne pas laisser chômer les filles, toutes les fois que l'occasion favorable s'en présentera? - Je le promets. ${ }^{21}$

Damit wird gleichsam ein Versprechen eingeholt, welches nicht allein eine große sexuelle Freizügigkeit der männlichen Besatzungen in der Neuen Welt beinhaltet, sondern zugleich auch eine männliche Unterwerfung der weiblich gegenderten Kolonien symbolisiert. In der Neuen Welt gelten folglich für die Männer andere Formen und Normen der Konvivenz, die ganz unverkennbar die männliche Potenz ins Zentrum jeglicher Geschlechterordnung rücken. Damit werden beim Übertritt von der einen in die andere Hemisphäre schon einmal klar die männlich zentrierten Unterwerfungswünsche fixiert. Es ist, als würde in diesem Akt auf einer symbolischen Ebene die Inbesitznahme einer neuen Welt, wie sie am Ausgang des 15. Jahrhunderts bei Columbus in einer genau festgehaltenen juristischen Vorgehensweise abgespult wurde, nun für die Geschlechterordnungen definiert. Wir sehen also, welche eminent politische und symbolische Bedeutung die Geschlechterbeziehungen im Reisebericht wie in der Wirklichkeit, einmal in die Neue Welt projiziert, besitzen.

Die Serie von Versprechen, die mit einem Schwur und einer ritualisierten Taufszene abgeschlossen wird, in der aus christlicher Sicht stets die Symbolik des Beginns eines anderen Lebens gegenwärtig ist, eröffnet eine Abfolge weiterer

21 Pernety, Antoine-Joseph: Journal historique d'un Voyage aux Iles Maloü̈nes en 1763 \& 1764, Bd. 1, S. $107 \mathrm{f}$. 
Lustbarkeiten, bei denen auch zwei paradiesische „Demoiselles Acadiennes“22 sowie allerlei neckische Spiele nicht fehlen dürfen, die sich in der Folge auf Bougainvilles Fregatte unter dem Kommando des bon-homme de la Ligne abspielen. Die Projektion männlich beherrschter sexueller Freizügigkeit auf die Tropen, auf die 'andere' Welt, zählt zum Kernbestand europäischer Bilderwelten von fernen Ländern. Blicken wir uns heute in den Katalogen der Veranstalter von Fernreisen in die Karibik oder nach Thailand um, so wird deutlich, dass derlei Traditionen keineswegs verloren gegangen sind.

So werden von europäischen Sexualphantasien geprägte Erörterungen über die Geschlechtlichkeit und das Geschlechtsleben der indigenen Bevölkerungen nicht von ungefähr auch in der Berliner Debatte über die Neue Welt eine wichtige (wenn auch hier nicht weiter verfolgte) Rolle spielen. Die Indianer werden dabei im Gegensatz zu den lasziven Indianerinnen, die sich nur so nach Europäern sehnen, zu impotenten Eunuchen degradiert, die es an sexueller Potenz mit den europäischen Männern niemals aufnehmen könnten.

All dies ist ein symbolischer Akt mit Folgen nicht allein in Bezug auf die Geschlechterordnungen. Innerhalb der narrativen Abfolge des Journal historique schärft die ausführliche Darstellung der Äquatortaufe die Aufmerksamkeit des Lesepublikums für die diskursive (und epistemologisch grundlegende) Andersartigkeit der 'anderen' Welt. Denn zwischen den Kapverden und der brasilianischen Küste quert die Fregatte nicht nur die Äquatoriallinie, sondern tritt mit ihrer Besatzung und ihren Passagieren symbolisch in eine andere Welt, in ein für die französische Besatzung neues Leben ein. Nichts bleibt, wie es war.

Entscheidend ist nicht, dass sich die Formen der Taufe von Nation zu Nation, aber auch von Schiff zu Schiff unterscheiden; ausschlaggebend ist vielmehr die Tatsache, dass dieses Baptême stets an Bord durchgeführt wird und ein verbrieftes Recht der Mannschaften darstellt. ${ }^{23}$ Dabei ist die Taufe nicht vorrangig an die Querung der Äquatoriallinie selbst, sondern an den Eintritt in die Zone der Wendekreise gebunden, wie Pernety abschließend erläutert:

Lorsque le Navire dans sa route ne doit pas passer la Ligne, mais seulement le Tropique, ceux des Equipages qui l'ont déjà passé, ne voulant pas perdre ce droit de tribut, se sont avisés de nommer le Tropique, le fils aîné du Bon-homme [de] la Ligne, héritier présomptif de ses droits. Ils jouent en conséquence, au passage du Tropique, la même farce que les autres sous l'Equateur. ${ }^{24}$

22 Ebda., S. 109.

23 Ebda., S. 111.

24 Ebda., S. 112. 
So stellen die Tropen als weltumspannender Bewegungs-Raum den 'eigentlichen’ dynamischen Kern europäischer Alteritätsvorstellungen dar. Tropen sind sie folglich in einem dreifachen Sinne: als Raum der Bewegungen (der Sonne zwischen den Wendekreisen) und als Bewegungsraum (der Europäer in ihren transozeanischen Verbindungen), zugleich aber auch als Tropen in einem rhetorisch-literarischen Sinne, in welchem die übertragenen, projizierten Bedeutungen vorherrschen und bestimmend sind. Wir werden auf diese Thematik noch mehrfach im Verlauf dieses dritten Teiles unserer Vorlesung zurückkommen.

Doch noch einmal zur Äquatorialtaufe in Antoine-Joseph Pernetys Reisebericht. Dass diese gerade auch religiöse Elemente karnevalisierende Szenerie in der Folge freilich ebenso auf andere 'Grenzlinien' übertragen wurde und wie etwa die rituelle 'Polartaufe' an Bord des deutschen Forschungsschiffes 'Polarstern' noch heute zeigen kann - auch in unseren Tagen an Bord vieler Schiffe gefeiert wird, vermindert keineswegs die anhand dieser rites de passage erkennbar werdende symbolische Bedeutung der Bewegungsfigur der Tropen. Denn diese abendländisch entworfenen Tropen sind Tropen der Verwandlung, der Metamorphose, in denen der Transfer von einer 'Alten' in eine 'Neue' Welt mit einer fundamentalen Transformation aller Sitten, Gewohnheiten und Lebensverhältnisse einhergeht. Die Neue Welt ist in den europäischen Reiseberichten des 18. Jahrhunderts eine andere Welt, die von der Alten Welt als klar geschiedene Gegen-Welt re-präsentiert wird.

Doch diese Andersheit der Tropen und des tropischen Amerika steht im europäischen Wissenschaftsdiskurs nur allzu oft im Zeichen einer fundamentalen Inferiorität. Die Todorov'sche Kippfigur lässt wieder einmal schön grüßen. Wie sehr sich in der zweiten Hälfte des 18. Jahrhunderts, während der zweiten Phase beschleunigter Globalisierung, auch die historischen Kontexte gewandelt haben mögen und an die Stelle der iberischen Mächte längst Frankreich und England als globalisierende Führungsnationen getreten sind: Jene Tropen, die aus europäischer Perspektive den Blick auf die Tropen bestimmen, finden sich weitgehend unverändert in den Schriften der philosophes der europäischen Aufklärung. Es ist diese Kontinuität unter allen kontextuellen Veränderungen, welche in den Beziehungen während der Phasen beschleunigter Globalisierung immer wieder beeindruckt. Denn immer wieder pausen sich bei allen Veränderungen und Verschiebungen dieselben menschlich-allzumenschlichen Verhaltensweisen durch.

Genau an dieser Stelle einer Zuordnung von Superiorität und Inferiorität entbrennt dann letztlich die Berliner Debatte um die Neue Welt. Ohne über einen längeren Zeitraum jemals Gehör in Europa $\mathrm{zu}$ finden, wehrten sich amerikanische Aufklärer wie der neuspanische Jesuit Francisco Javier 
Clavijero $^{25}$ vehement und mit guten Gründen dagegen, in der Nachfolge Buffons von der zeitgenössischen europäischen Wissenschaft zum inferioren Anderen Europas abgestempelt zu werden. Jenseits ihrer Fülle aber erscheint die Tropenwelt Amerikas in den Augen der meisten europäischen Philosophen als eine Falle, die ganz im Zeichen ihrer behaupteten grundlegenden Inferiorität eine von der 'eigenen' grundsätzlich geschiedene ‘andere' Welt konfiguriert. Und diese Welt, so sahen es die meisten europäischen philosophes der Aufklärung, musste inferior sein.

So verweist die Beschreibung der am 10. November 1763 erfolgten Querung des Äquators im Journal historique von Antoine-Joseph Pernety bereits voraus auf grundsätzliche Auseinandersetzungen, die im Verlauf der wenigen Jahre nach dieser Reise ausgebrochenen Berliner Debatte um die Neue Welt geführt werden sollten. Denn gerade die These von der fundamentalen Schwäche und Unterlegenheit der Neuen Welt hatte in der europäischen Aufklärungsliteratur Bilder erzeugt, die in den 1768 und 1769 in Berlin erschienenen Recherches philosophiques sur les Américains ${ }^{26}$ des Cornelius de Pauw im Zeichen der Degenerationsthese die Stufe einer teilweise geradezu apokalyptischen Bilderwelt erreichten. Wie konnte es zu einer derartigen Zuspitzung kommen?

Für den 1739 in Amsterdam geborenen und 1799 in Xanten verstorbenen Kleriker de Pauw war es evident, dass sich von den Tropen ausgehend Krankheiten und Epidemien wie Syphilis oder Gelbfieber über den gesamten Erdball ausbreiten würden und das menschliche Leben auf dem Planeten in seinem Fortbestand gefährden mussten. Seiner Vision kann man folglich deutliche apokalyptische Züge nicht absprechen. Er hatte begriffen, dass seine Zeit die Zeit eines erheblich intensivierten Austausches war, eine Zeit, in der jener Prozess de longue durée, den wir heute 'die' Globalisierung nennen, wieder erheblich an Fahrt aufgenommen hatte. Genau hier lag sein Ansatzpunkt.

Doch greifen wir der konkreten Abfolge der Ereignisse rund um die Berliner Debatte um die Neue Welt nicht vor. Darin mochte Antoine-Joseph Pernety in seiner Rede vom 7. September 1769 vor jener Berliner Académie des Sciences \&

25 Vgl. das zunächst im italienischen Exil in italienischer Sprache erschienene Werk von Clavijero, Francisco Javier: Storia Antica del Messico. 4 Bde. Cesena: Gregorio Biasani 1780. Vgl. hierzu allgemein auch Ette, Ottmar: Archeologies of Globalization. European Reflections on Two Phases of Accelerated Globalization in Cornelius de Pauw, Georg Forster, GuillaumeThomas Raynal and Alexander von Humboldt. In: Culture \& History Digital Journal (Madrid) I, 1 (June 2012) <http://dx.doi.org/10.3989/chdj.2012.003> (20 S.).

26 Vgl. Pauw, Cornelius de: Recherches philosophiques sur les Américains, ou Mémoires intéressants pour servir à l'Histoire de l'Espèce humaine. 2 Bde. Berlin: Chez Georges Jacques Decker, Imp. du Roi 1768-1769. 


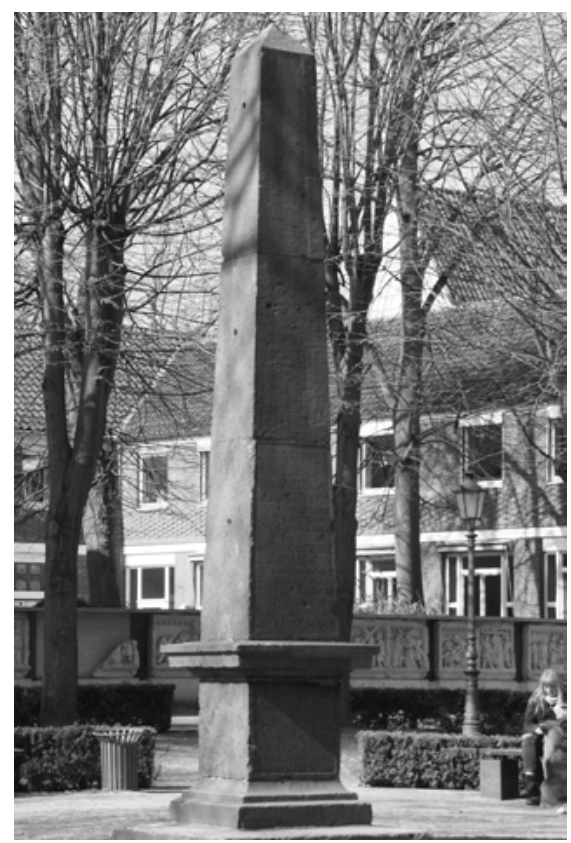

Abb. 67: Obelisk zu Ehren Cornelius de Pauws (Amsterdam, 1739 - Xanten, 1799) in Xanten.

Belles-Lettres, deren Mitglied er war, auch eine dezidierte Gegenposition gegen Cornelius de Pauw entwickeln, so zeigte sich gleichwohl, dass in dieser 'Berliner Debatte' über die Neue Welt, ${ }^{27}$ die weit über die Grenzen Preußens und Europas hinaus wahrgenommen wurde, die Position de Pauws und damit eine Position obsiegte, in der die 'Neue Welt', die auch geologisch jünger als die Alte und folglich viel später erst aus den Wassern emporgestiegen sei, als Ort einer prinzipiellen, von Anfang an gegebenen Inferiorität schlechthin stigmatisiert wurde. Diese ebenso radikale wie (in der Tradition Buffons) populäre These stammte wohlgemerkt von einem Autor, der zu keinem Zeitpunkt Europa jemals verlassen oder gar die von ihm dargestellte Hemisphäre Amerikas je betreten hatte. Wir haben es hier also erneut mit einer Variante des Konflikts zwischen Reisenden und Daheimgebliebenen und damit dem Verhältnis zwischen Reisen und Schreiben zu tun.

27 Vgl. zur Berliner Debatte Ette, Ottmar: Archeologies of Globalization. European Reflections on Two Phases of Accelerated Globalization in Cornelius de Pauw, Georg Forster, GuillaumeThomas Raynal and Alexander von Humboldt. In: Culture \& History Digital Journal (Madrid) I, 1 (June 2012) <http://dx.doi.org/10.3989/chdj.2012.003> (20 S.). 
Gerade im zweiten Band seiner Recherches philosophiques steigerte sich de Pauw im Kontext der Berliner Debatte zu Äußerungen, in denen die Europäer als Krönung des Menschengeschlechts erschienen und zugleich die Tropen und deren Bewohner völlig inferiorisiert, ja letztere aus dem Menschengeschlecht geradezu ausgeschlossen wurden. So heißt es 1769 bei de Pauw von der Spezies Mensch in scheinbar weltweitem Vergleich:

Le véritable pays où son espèce a toujours réussi \& prospéré, est la Zone tempérée septentrionale de notre hémisphère: c'est le siége [sic!] de sa puissance, de sa grandeur, \& de sa gloire. En avançant vers le Nord, ses sens s'engourdissent \& s'émoussent: plus ses fibres \& ses nerfs gagnent de solidité \& de force, par l'action du froid qui les resserre; \& plus ses organes perdent de leur finesse; la flamme du génie paroît s'éteindre dans des corps trop robustes, où tous les esprits vitaux sont occupés à mouvoir les ressorts de la structure \& de l'économie animale. [...] Sous l'Equateur son teint se hâle, se noircit; les traits de la physionomie défigurée révoltent par leur rudesse: le feu du climat abrége [sic!] le terme de ses jours, \& en augmentant la fougue de ses passions, il rétrécit la sphère de son ame: il cesse de pouvoir se gouverner lui-même, et ne sort pas de l'enfance. En un mot, il devient un Nègre, \& ce Nègre devient l'esclave des esclaves.

Si l'on excepte donc les habitants de l'Europe, si l'on excepte quatre à cinq peuples de l'Asie, \& quelques petits cantons de l'Afrique, le surplus du genre humain n'est composé que d'individus qui ressemblent moins à des hommes qu'à des animaux sauvages: cependant ils occupent sept à huit fois plus de place sur le globe que toutes les nations policées ensemble, \& ne s'expatrient presque jamais. Si l'on n'avoit transporté en Amérique des Africains malgré eux, ils n'y seroient jamais allés: les Hottentos ne voyagent pas plus que les Orangs [...]. ${ }^{28}$

Das ist hier fürwahr starker Tobak! Cornelius de Pauw wusste zuzuspitzen. Der allergrößte Teil der Menschheit wird in diesen einst berühmten Passagen gleichsam tropikalisiert. Tropikalisierung meint hier unübersehbar Inferiorisierung und zugleich eine Animalisierung, welche jene Debatte um die Grenze zwischen Mensch und Tier wieder aufnahm, die bereits in der ersten Phase beschleunigter Globalisierung zum Kernbestand der europäischen Diskussionen über den mundus novus gehört hatte. Dass damit auch eine Legitimation der Sklaverei verbunden war, wird an den soeben angeführten Passagen überdeutlich.

Die Buffons Histoire naturelle zuspitzenden und damit wissenschaftlich im Verständnis des 18. Jahrhunderts legitimierten Äußerungen des holländischen philosophe, der niemals in Amerika, dafür aber zweimal für einige Monate am preußischen Hof in Berlin und Potsdam weilte, entwerfen einen Gegensatz zwischen den gemäßigten Zonen insbesondere Europas einerseits und den Tropen Afrikas, Asiens und Amerikas andererseits, den als ein Zeugnis des Eurozentrismus zu bezeichnen wohl eher ein Euphemismus wäre. Wie kaum ein anderer

28 Pauw, Cornelius de: Recherches philosophiques sur les Américains, a. a.O., Bd. II, S. 68 f. 
europäischer Autor des 18. Jahrhunderts verstand es der Verfasser der Recherches philosophiques sur les Américains, seine philosophischen Untersuchungen zur indigenen Bevölkerung Amerikas ebenso polemisch wie protorassistisch auszuformulieren. Eben dies machte ihn unter seinen europäischen Zeitgenossen berühmt und ließ ihn weltweit zum Inbegriff des hassenswerten Europäers werden.

Zugleich aber wird in diesem Werk, das - wie noch zu zeigen sein wird auch die zerstörerische Kraft europäischer Wissenschaft insbesondere in der zweiten Phase beschleunigter Globalisierung anprangerte, deutlich, dass die klimatologische Argumentation eine Welt zwischen den Wendekreisen abwertet, die im Gegensatz zu Europa transkontinental und weltumpsannend strukturiert ist. De Pauw tat dies zu einem Zeitpunkt, als die ökonomische Ausplünderung der Tropen insbesondere von England und Frankreich aus auf einen neuen Höhepunkt geführt und ebenso transkontinental wie transareal, unterschiedlichste Kulturräume der Tropen miteinander verknüpfend, organisiert wurde. Frankreich und England waren die europäischen Führungsnationen, was die kapitalistische Inwertsetzung einer sklavenbetriebenen Plantagenwirtschaft in den Tropen weltweit anging. Dies gilt es gerade an dieser Stelle, allem Kopfschütteln zum Trotz, nicht zu vergessen.

Längst bildeten die Tropen eine intern vernetzte, aber kolonialistisch von außen kontrollierte transareale Struktur, die von Europa aus als komplementärer Ergänzungsraum funktionalisiert und gezielt globalisiert wurde. Die Verwandlung der Tropen in planetarisch ausgeweitete, dependente Gebiete, die freilich noch immer an den Tropen der Verwandlung partizipierten, wurde in der zweiten Phase beschleunigter Globalisierung abgeschlossen. Spätestens ab diesem Zeitpunkt begannen die Traurigen Tropen ${ }^{29}$ eines Claude Lévi-Strauss konkrete historische Gestalt anzunehmen. Wir werden im Rahmen der Vorlesung darauf zurückkommen. Doch greifen wir nicht vor.

Gleich zu Beginn seiner am 7. September 1769 vor der Berliner Akademie vorgetragenen und im Folgejahr veröffentlichten Dissertation sur l'Amérique et les Américains, contre les Recherches philosophiques de Mr. de $P^{* * * 30}$ baute Antoine-Joseph Pernety eine Frontstellung gegen die Thesen de Pauws auf, deren Strategie wir bereits an ihrem Beginn leicht erkennen können. Dazu genügt es, sich mit dem Duktus der Ausführungen Pernetys gleich am Anfang seiner 'Dissertation' zu beschäftigen:

29 Vgl. Lévi-Strauss, Claude: Tristes Tropiques. Paris: Librairie Plon 1955.

30 Vgl. hierzu auch Gerbi, Antonello: La Disputa del Nuovo Mondo, S. 120-125. 
Monsieur de P. vient de mettre au jour un Ouvrage sous ce titre, Recherches Philosophiques sur les Américains. Il s'efforce d'y donner l'idée la plus désavantageuse du nouveau Monde \& de ses habitants. Le ton affirmatif \& décidé avec lequel il propose et résoud ses questions; le ton d'assurance avec lequel il parle du sol \& des productions de l'Amérique, de sa température, de la constitution corporelle \& spirituelle de ses habitants, de leurs moeurs \& de leurs usages, enfin des animaux; pourroient faire croire qu'il a voyagé dans tous les pays de cette vaste étendue de la terre; qu'il a vêcu [sic!] assez longtemps avec tous les peuples qui l'habitent. On seroit tenté de soupçonner, que, parmi les Voyageurs, qui y ont fait de longs séjours, les uns nous ont conté des fables, ont travesti la vérité par imbécillité, ou l'ont violée par malice. ${ }^{31}$

Dom Pernety, der - wie bereits dargestellt - im selben Jahr 1769 ebenfalls in Berlin und in französischer Sprache seinen zweibändigen Reisebericht vorlegte, spielte hier von Beginn an die Karte dessen, der als Augenzeuge jene Länder bereiste, die sein Widersacher Cornelius de Pauw ungeachtet des Grundtons größter Selbstsicherheit, mit der er nicht zuletzt auch die Berichte von Reisenden in der Tat einer pauschalen Kritik unterwarf, niemals selbst zu Gesicht bekam. Es ist die Karte des Reisenden gegen den Daheimgebliebenen, die Karte dessen, der alles von eigenem Augenschein her kennt, gegen denjenigen, der nur alles vom Hörensagen weiß, ohne es selbst überprüft zu haben.

Demgegenüber stellte sich Pernety selbst, der als Akademiemitglied zurecht auf eine positive Aufnahme seiner Überlegungen durch die Berliner Akademie hoffen durfte, in den ihm sicherlich bekannten Zusammenhang jener Forderung nach einem philosophe voyageur, welche Rousseau in seinem Discours sur l'origine et les fondements de l'inégalité parmi les hommes - wie wir gesehen hatten - erhoben hatte. Pernety hatte, so schien es, die guten Argumente auf seiner Seite.

Daher betonte er bereits auf den ersten Zeilen seiner 'Préface', dass er sich der Welt Amerikas nicht allein als Leser angenähert habe: „J'avois lu \& relu quantité de rélations de l'Amérique; j'avois vu de mes propres yeux la plupart des choses, qui y sont rapportées. “32 Und es habe ihn sehr erstaunt, all die Dinge, die er mit eigenen Augen gesehen habe, verworfen oder verkleidet $\mathrm{zu}$

31 Ich zitiere nach der von Cornelius de Pauw in den dritten Band seiner 1770 erschienenen dreibändigen Ausgabe der Recherches philosophiques aufgenommenen Fassung; vgl. Pernety, Antoine-Jseph: Dissertation sur l'Amérique et les Naturels de cette partie du Monde. In: Pauw, Cornelius de: Recherches philosophiques sur les Américains, ou Mémoires intéressants pour servir à l'Histoire de l'Espèce humaine par Mr. de P. Nouvelle Edition, augmentée d'une Dissertation critique par Dom Pernety; \& de la Défense de l'Auteur des Recherches contre cette Dissertation. 3 Bde. Berlin: Decker 1770, Bd. 3, S. $7 \mathrm{f}$.

32 Ebda., S. $4 \mathrm{f}$. 
sehen: „voir contredites, ou travesties par Mr. de P.“33 Wie bei Rousseau ist auch bei Pernety das eigene Sehen und damit der Gesichtssinn des reisenden Philosophen von entscheidender Bedeutung für eine fundierte Beurteilung. Pernety fühlte sich seinem Gegner in der von ihm angezettelten Debatte haushoch überlegen.

Auch an anderen Stellen der Dissertation lässt sich unschwer erkennen, dass die Gedankenwelt Rousseaus in den Argumentationen Pernetys eine nicht zu unterschätzende Rolle spielt. Dies betrifft keineswegs nur den von Rousseau ererbten Naturbegriff. So kehrt Pernety de Pauws Bild von den schwachen, geistig wie körperlich unterlegenen und unmännlichen (da bartlosen) Indianern um in ein genau gegenläufiges Bild, besitze die indigene Bevölkerung doch „une santé ferme, vigoureuse, une vie qui passe ordinairement les bornes de la nôtre“. ${ }^{34}$ Dem starken, langlebigen Naturmenschen (im Stile eines Rousseau) eignet aber auch ein wacher Geist: „un esprit sain, instruit, éclairé \& guidé par une philosophie vraiment naturelle, \& non subordonnée comme la nôtre, aux préjugés de l'éducation; une ame noble, courageuse, un coeur généreux, obligeant: que faut-il donc de plus à Mr. de P. pour être véritablement hommes?“35

Der Naturmensch à la Rousseau ist bei Pernety allgegenwärtig. Die Diskussion des Mensch-Tier-Gegensatzes nutzt der Abbé geschickt, um neben der schon früh in seinen Text eingefügten und oft wiederholten Erwähnung amerikanischer Hochkulturen wie der Incas zugleich seine Gegen-These von der Superiorität des homme naturel, also der Urbevölkerung Amerikas, gegen de Pauw vorteilhaft in Stellung zu bringen. Die Vertreter einer „philosophie vraiment naturelle“ dürfe man keinesfalls als Wilde, als „Sauvages“, bezeichnen, müssten sich doch eher die Europäer ein derartiges Etikett gefallen lassen: „puisqu'en effet nos actions sont contraires à l'humanité, ou du moins à la sagesse qui devroit être le guide des hommes, qui se piquent d'être plus éclairés qu'eux“. ${ }^{36}$ Man spürt sehr deutlich, wohin die Argumentation des Franzosen zieht.

Mokiert sich hier Pernety über die von seinem Gegner in der Berliner Debatte vorgetragene „Belle leçon dictée par les lumieres de la pure raison“, 37 so beklagt er im gleichen Atemzug das Unglück und die Unruhe eines Teils des Menschengeschlechts, das sich über alle anderen Teile erhaben glaube. Die Gesellschaft derer, die man als 'Wilde' bezeichne, sei hingegen eine Gemeinschaft, die auf einem Contrat social, auf einem Gesellschaftsvertrag ganz im Sinne Rousseaus gründe:

33 Ebda., S. 5.

34 Ebda., S. 114.

35 Ebda., S. $114 \mathrm{f}$.

36 Ebda., S. 115.

37 Ebda. 
J'avoue que nous sommes faits les uns pour les autres, \& que de cette dépendance mutuelle résulte tout l'avantage de la société. Mais la premiere intention de cette union, ou Contract Social; a été d'obliger tous les contractants à se prêter des secours mutuels, \& non de laisser tout usurper aux uns; de les authoriser même dans leurs usurpations \& de laisser manquer de tout aux autres. ${ }^{38}$

Damit wird deutlich, dass Antoine-Joseph Pernety der vernichtenden Einschätzung der indigenen Bevölkerung durch Cornelius de Pauw nicht nur den starken Naturmenschen und Naturphilosophen auf der individuellen Ebene, sondern auch den Menschen der Gemeinschaft, den Menschen des Contrat social, auf der kollektiven Ebene geradezu idealtypisch - und in jedem Falle idealisierend - gegenüberstellt. Er weiß sich hier in bester philosophischer Gesellschaft. aber waren diese Argumente gerade mit Blick auf die sozialen Strukturen indigener Gesellschaften wirklich stichhaltig? Waren sie mehr als das genaue Gegenteil dessen, was sein aus Holland stammender Widersacher behauptet hatte?

Man darf in dieser Argumentationsweise sehr wohl eine Schwäche der Strategie Pernetys erkennen, stellt er den negativ eingefärbten Bildern de Pauws doch allzu oft einfach positiv eingefärbte Gegen-Bilder entgegen. Diese gleichsam inverse Darstellung macht seine eigene Argumentation abhängig von jener seines Gegners, da sich Pernety immer wieder darauf beschränkt, die negativen Vorzeichen auf allen Ebenen in positive zu verwandeln und daraus eine Polemik zu entfachen, die doch ein wenig kurz greift. Darunter aber leidet die Eigenständigkeit seiner Beobachtungen wie die Originalität und Beweglichkeit seiner Dissertation, die zeitweise zum reinen Gegen-Diskurs verkommt. Wir erkennen hierin eine Schwäche, die sein Gegner eiskalt ausnutzen sollte.

Diese Argumentationslinie, das genaue Gegenteil dessen zu behaupten, was de Pauw in seinen Recherches philosophiques vorstellte, prägt auch die 'Seconde Partie' der Pernety'schen Akademierede, die sich schon auf den ersten Zeilen von dem abwendet, was man „Herrn de P. zufolge glauben müßte“. ${ }^{39}$ Denn dieser Teil der Erde sei weltweit der beste, das Land sei äußerst fruchtbar, die Bäume überladen mit Früchten, wie er selbst im Garten des Gouverneurs von Montevideo mit eigenen Augen gesehen habe ${ }^{40}$ - und überhaupt könne man diesen wunderbaren Erdteil am besten mit dem Irdischen Paradies ${ }^{41}$ und mit den Gärten des Goldenen Zeitalters vergleichen: eine Welt der Wunder und

38 Ebda., S. 115f.

39 Ebda., S. 32.

40 Ebda.

41 Ebda., S. 35. 
einer Fülle, wie sie einst ein Vergil besungen habe. ${ }^{42}$ Auch ein Columbus hätte nicht besser die Vorzüge Amerikas vor Europa besingen können!

Der französische Abbé war nun nicht mehr zu halten. Würde man de Pauw folgen, so müsste man die amerikanische Hemisphäre als eine „terre maudite“ begreifen, ${ }^{43}$ in Wirklichkeit aber habe sich Pernety selbst davon überzeugen können, dass in Amerika „le principe de vie“, folglich das Lebensprinzip und wohl auch die Lebenskraft, wesentlich stärker ausgeprägt seien als in Europa. ${ }^{44}$ Nichts von dem, was de Pauw in seinen Recherches philosophiques sur les Américains behauptet, bleibt in Pernetys Gegen-Diskurs bestehen. Aber war dies eine intelligente Diskursstrategie?

Zweifellos war es ebenso zutreffend wie klug, gegen die einseitigen Anschuldigungen de Pauws, der amerikanische Kontinent habe den Rest der Welt mit Epidemien und Krankheiten - allen voran der Syphilis - überzogen, mit guten Gründen auf eine wechselseitige Globalisierung von Krankheiten aufmerksam zu machen, wie sie in der Tat mit der ersten Phase beschleunigter Globalisierung einhergegangen war.

Doch Pernety hatte sich darauf festgelegt, stets das genaue Gegenteil dessen zu behaupten, was de Pauw zu Protokoll gegeben hatte. So kam er nicht umhin, auch auf dem Gebiet der Krankheiten und Epidemien Amerika und seine Bewohner in ein wahres Paradies auf Erden zu verwandeln. Die Bewohner Amerikas würden keineswegs von unendlich vielen und schrecklichen Krankheiten gepeinigt, sondern seien vielmehr als überaus gesund und robust anzusehen:

Soit par l'exercice, soit par la constitution de leur tempérament, ils sont fort sains, exempts de paralysie, d'hydropilie, de goutte, d'héthysie, d'asthme, de gravelle, de pierre; maladies dont la Nature qui a tant donné à notre continent, a bien encore voulu nous favoriser. Elle avoit cependant laissé la pleuresie au Canada; \& nous leur avons porté la petite vérole. Les Américains nous ont communiqué la leur par droit d'échange \& de commerce. ${ }^{45}$

Gegen die gewiss nicht nur von de Pauw vertretene These von der Degenerierung des Menschengeschlechts in den Tropen der Neuen Welt setzt Pernety eine hemisphärische Konstruktion, die den amerikanischen (d.h. indigenen) Menschen vom hohen Norden bis zum tiefen Süden unter Verweis auf die unterschiedlichsten Reisenden in das glanzvollste Licht rückt. So heißt es von der indigenen Bevölkerung des gesamten Kontinents:

42 Ebda., S. 36.

43 Ebda., S. 43.

44 Ebda., S. 42.

45 Ebda., S. 48. 
Si nous remontons du septentrion jusqu'à l'extrémité méridionale du nouveau Continent, tous les peuples que nous rencontrons sur notre route, offrent des hommes bien constitués. Tels sont, si nous en croyons Vincent le Blanc \& les autres Voyageurs, les Mexicains, les Brésiliens, les Péruviens, ceux du Paraguai, du Chili \& enfin les Patagons. ${ }^{46}$

Dabei zeichneten sich nicht nur einzelne Völker, sondern alle Amerikaner ebenso durch eine den Europäern gegenüber weit größere und ehrlichere Gastfreundschaft aus, ja legten Tugenden an den Tag, die man zu bewundern nicht umhin könne:

Sans avoir de Licurgues pour Législateurs, les Caraibes, \& en général tous les Américains respectent infiniment les vieillards, les écoutent avec attention, déferent aux sentiments des anciens, \& se réglent sur leurs volontés Ils sont naturellement francs, véridiques, \& ont donné dans tous les temps des marques de candeur, de courtoisie, d'amitié, de générosité, \& de gratitude. Ceux qui les ont pratiqué long-temps leur rendent plus de justice que Mr. de P. Si l'on trouve aujourd'hui chez eux le mensonge, la perfidie, la trahison, le libertinage, \& plusieurs autres vices, on doit s'en prendre aux pernicieux exemples des Européans, \& aux mauvais traitements que ceux ci ont exercés contre eux. A chaque page des rélations, on voit combien ceux de l'ancien Continent ont fait valoir dans le nouveau, l'art qu'ils savent si bien, de tromper vilainement. ${ }^{47}$

Pernety entwirft in diesen Formulierungen ein Portrait der indigenen Bevölkerung, wie es harmonischer und sittenstrenger nicht sein könnte. Wir haben es ihm zufolge bei den Amerikanern, also der Urbevölkerung Amerikas, mit wahren Vorbildern an Tugend, Großzügigkeit und Wahrhaftigkeit zu tun - eine wahre Modellgesellschaft, die ihren Schatten auf die europäischen Gesellschaften wirft.

Angesichts eines derartigen Sittengemäldes, das in einem scharfen Kontrast den robusten und guten Naturmenschen vom nicht nur verdorbenen, sondern weit mehr noch die anderen Völker verderbenden Europäer absetzt, verwundert es nicht, dass Pernety die Bewohner Amerikas pauschal auch zu den glücklicheren Menschen erklärt. ${ }^{48}$ Die wenig nuancierende Argumentationsstrategie, die es alleine darauf anlegt, de Pauws Behauptungen durch Gegen-Behauptungen zu widerlegen, affiziert letztlich weite Teile der Dissertation, die sich in diesen langen Passagen weder empirisch noch epistemologisch den Ausführungen de Pauws als überlegen erweist. Im Gegenteil: Pernetys argumentative Linie erweist sich auf Dauer als reichlich ermüdend.

Mit der von Pernety immer wieder vorgetragenen Frage nach den Patagoniern greift das Mitglied der Berliner Akademie eine Problematik auf, die auch Jean-Jacques Rousseau nicht von ungefähr erwähnt hatte: „sans oublier les

46 Ebda., S. $49 \mathrm{f}$.

47 Ebda., S. 81-83.

48 Ebda., S. 87. 
Patagons vrais ou faux“. ${ }^{49}$ Zweieinhalb Jahrhunderte nach Pigafettas ersten Berichten von Riesen in Patagonien sollten derlei Behauptungen nun endlich einer wissenschaftlichen Erforschung und abschließenden Bewertung zugeführt werden, um ihren Wahrheitsgehalt zu überprüfen.

Nicht umsonst hatte Pernety seinem Reisebericht eine Relation sur les $\mathrm{Pa}$ tagons beigefügt, die er publikumswirksam bereits in den Titel seines Journal historique aufgenommen hatte. Und hatte nicht Pierre Moreau de Maupertuis höchstselbst, der Präsident der Berliner Akademie, zu den riesenhaften Patagoniern verlauten lassen, dass man vernünftigerweise nicht mehr an ihrer Existenz zweifeln könne ${ }^{50}$ Es dürfte kaum überraschen, dass er de Pauws Behauptung, bei der Rede von den Menschen mit riesemhaften Wuchs in Patagonien handele es sich um von europäischen Reisenden erflunkerte Fabelwesen, nicht nur die teilweise Manipulation von Quellen vorwarf, ${ }^{51}$ sondern in ganz grundsätzlicher Manier entgegentrat:

Je ne conçois pas comment Mr. de P. a entrepris d'anéantir l'existence des Patagons Geánts. En raisonnant suivant sa méthode philosophique, rien n'étoit plus capable que cette d'existence, de prouver à ses yeux, la dégradation \& la dégénération de la race humaine en Amérique. ${ }^{52}$

Doch gerade an dieser Stelle wird deutlich, wie sehr Pernety mit seinem nicht selten sehr mechanisch wirkenden Bemühen, de Pauw in allen Punkten zu widerlegen, über sein Ziel hinausschoss und die Legitimität und Autorität seines eigenen Diskurses dabei beschädigte. Dies dürfte entscheidend zu dem unbestreitbaren Faktum beigetragen haben, dass sich die von Pernety inkriminierte méthode philosophique des Cornelius de Pauw in der Berliner Debatte, also in jener zunächst von Berlin ausgehenden europäischen Phase des Disputs um die Neue Welt, letztlich durchzusetzen vermochte. Denn das allgemeine Publikum schenkte den (pseudo-)kritischen Anmerkungen und Infragestellungen Cornelius de Pauws wesentlich größeren Glauben als einer Sichtweise, welche die den Europäern zumindest militärisch und zivilisatorisch ganz offenkundig unterlegenen Indigenen in glückliche Naturmenschen umstilisierte. Die Vorgehensweise de Pauws, die Argumente seiner Gegner kritisch

49 Rousseau, Jean-Jacques: Discours sur l'origine et les fondements de l'inégalité parmi les hommes, S. 213.

50 Vgl. hierzu Moureau, François: L’Amérique n'a aucun avenir: les idées 'philosophiques' de Cornelius De Pauw. In: Travaux de Littérature (Genève) 24 (2011), S. 66.

51 Pernety, Antoine-Joseph: Dissertation sur l'Amérique et les Naturels de cette partie du Monde, S. 68.

52 Ebda., S. 51. 
einzubeziehen, bevor er sie zerstörte, entsprach wesentlich mehr jener kritischen aufklärerischen Diktion, welche das europäische Publikum im Jahrhundert der Aufklärung goutierte.

Worin aber bestand diese Methode des Cornelius de Pauw genauer? Und welche Folgen hatte sie für ein Verständnis der unterschiedlichsten Phänomene der Globalisierung aus einer europäischen Perspektive im Zeitraum vor jenem Alexander von Humboldt, der genau eine Woche nach der Rede Pernetys vor der Berliner Akademie nach eigenem Bekunden im Zeichen eines Kometen in der preußischen Hauptstadt das Licht der Alten Welt erblickte?

Noch bevor wir uns genauer mit der Methode des holländischen Abbé beschäftigen, gilt es eines festzuhalten: Cornelius de Pauw ist ein Denker der Globalität und sein Werk eine Antwort auf jene zweite Phase beschleunigter Globalisierung, ${ }^{53}$ welche die zweite Hälfte des 18. Jahrhunderts bis deutlich über die Jahrhundertwende hinaus umfasst. Einheit und Aufteilung des Planeten stellen Problematiken dar, die de Pauws gesamtes Werk über die Alte wie die Neue Welt durchziehen.

Dabei begreift der Autor der Recherches philosophiques sur les Américains ganz im Sinne der Naturgeschichte Buffons diese 'Neue' Welt auch als eine geologisch wie geomorphologisch jüngere, neuere Welt. Seine Ausführungen hierzu entsprechen dem Forschungsstand eines Buffon und bilden geradezu 'klassische' Formulierungen der Novität der westlichen Hemisphäre:

Le nombre presqu'infini de lacs \& de marécages dont les Indes occidentales sont couvertes, n'avoit pas été formé uniquement par les eaux fluviales extravasées, ni par les brouillards attirés par les montagnes et les forêts: ces lacs paraoissoient être des dépôts d'eaux qui n'avoient pu encore s'écouler des endroits jadis noyés par une secousse violente, imprimée à toute la machine du globe terraquée: les nombreux volcans des Cordillieres \& des rochers du Mexique, les tremblements qui ne cessent jamais dans l'une ou dans l'autre branche des Andes, prouvent que la terre n'y est pas encore en repos de nos jours. ${ }^{54}$

Der gesamte Planet bildet mithin eine Einheit, jene 'Maschine des Globus', die sich freilich naturhistorisch gesehen gemäß dieser Theorie gleichzeitig in erdgeschichtlicher Ungleichzeitigkeit befindet. Denn während es in der Alten Welt längst zu einer gewissen Beruhigung (,repos“) gekommen ist, brodelt und schüttelt es in den Gebirgen der Neuen Welt, wo die Landmassen sich erst

53 Vgl. hierzu ausführlicher Ette, Ottmar: Weltbewußtsein. Alexander von Humboldt und das unvollendete Projekt einer anderen Moderne. Weilerswist: Velbrück Wissenschaft 2002, S. 25-27; sowie (ders.): TransArea - eine literarische Globalisierungsgeschichte, S. 14-18.

54 Pauw, Cornelius de: Recherches philosophiques sur les Américains, ou Mémoires intéressants pour servir à l'Histoire de l'Espèce humaine, Bd. 1, S. 102. 
wesentlich später aus dem Wasser gehoben hätten. 'Alt' steht 'neu' gegenüber. Und das 'Alte' ist dem 'Neuen' überlegen. Denn das Neue ist weit davon entfernt, zur Ruhe gekommen zu sein: Es formt sich ständig um und kann noch nicht jene Sicherheit ausstrahlen, die zur Entfaltung einer wahren Zivilisation notwendig ist. Zumindest gemäß der Thesen eines Cornelius de Pauw.

Die Maschine des Erdkörpers ist zwar durchaus eine einzige, spaltet sich aber in zwei Hemisphären auf, die in ihrer Gegensätzlichkeit bis in unsere Tage fortbestehen würden. Die Unterscheidung und Scheidung zweier Hemisphären bildet die grundsätzliche diskursive Setzung der Recherches. Der Gegensatz zwischen Alter und Neuer Welt ist damit erdgeschichtlich oder geologisch fundiert.

Diese Scheidung ist zunächst zwar rationaler, aber nicht relationaler Natur. Auf diesen beiden Ebenen, jener der Zweiteilung der Erde wie der NichtRelationalität ihres Verhältnisses, sind die Unterschiede zwischen den Sichtweisen de Pauws und Pernetys nicht sehr erheblich. Beide stimmen in der grundlegenden Einschätzung überein, dass sich beide Hemisphären als schroffe Gegensätze und damit unvermittelt gegenüberstehen. Nur ihre jeweils negative oder positive Attribuierung unterscheidet sich grundlegend voneinander.

Dem fundamentalen Argumentationsschema einer Zweiteilung, ja mehr noch einer Gegensätzlichkeit der beiden Hemisphären folgen nicht allein die naturhistorischen, sondern auch die kulturhistorischen Überlegungen der Recherches philosophiques sur les Américains. Gerade hierin ist ihr Biss und ihre Bissigkeit zu erblicken - und auch die Gründe hierfür, warum die $R e$ cherches de Pauws in Europa so populär wurden. Dabei machte Cornelius de Pauw von Beginn an deutlich, welches für ihn jenes historische Ereignis war, das aus seiner Sicht die Geschichte des Planeten am nachhaltigsten geprägt und gleichsam die naturhistorische Differenz zwischen Alter und Neuer Welt am stärksten hervorgetrieben und in den kulturgeschichtlichen Bereich deterministisch übersetzt hatte. Auch an dieser Stelle sind seine Formulierungen über die kulturgeschichtliche Bedeutung des Descubrimiento geradezu klassisch geworden:

Il n'y a pas d'evénement plus mémorable parmi les hommes, que la Découverte de l'Amérique. En remontant des temps présents aux temps les plus reculés, il n'y a point d'evénement qu'on puisse comparer à celui là; \& c'est sans doute, un spectacle grand \& terrible de voir une moitié de ce globe, tellement disgraciée par la nature, que tout y étoit ou dégéneré, ou monstrueux.

Quel Physicien de l'Antiquité eut jamais soupçonné qu'une même Planète avoit deux Hémisphères si différents, dont l'un seroit vaincu, subjugué \& comme englouti par l'autre, dès qu'il en seroit connu, après un laps de siécles qui se perdent dans la nuit \& l'abyme des temps? 
Cette étonnante révolution qui changea la face de la terre \& la fortune des Nations, fût absolument momentanée, parce que par une fatalité presqu'incroiable, il n'existoit aucun équilibre entre l'attaque et la défense. Toute la force \& toute l'injustice étoient du côté des Européens: les Américains n'avoient que de la foiblesse: ils devoient donc être exterminés \& exterminés dans un instant. ${ }^{55}$

Damit ist die Zweiteilung der Welt nicht allein naturhistorisch, sondern auch kulturhistorisch und zivilisatorisch ein für alle Mal begründet und gleichsam 'natürlich' legitimiert. Der nicht umsonst an Jesuitenkollegs in Lüttich und Köln ausgebildete und überdies an der für außereuropäische Fragen bestens ausgestatteten Göttinger Universität eingeschriebene Cornelius oder Corneille de Pauw ${ }^{56}$ entfaltete in den wie stets bei ihm scharf konturierten Wendungen seines französischsprachigen Werkes eine Gegensätzlichkeit, welche die Opposition von 'alter' und 'neuer' Welt nun auf jene von 'Europäern' und 'Amerikanern' übertrug. Zwischen beiden Gruppen könnten die Gegensätze und die Widersprüche nicht größer und wirkungsvoller sein. Wir alle sind Bewohner einer Welt, die schroffer zweigeteilt kaum vorstellbar sein könnte. So verbindet uns nichts als die Tragik einer seit der Entdeckung Amerikas gemeinsam geteilten dramatischen Geschichte von Sieg und totalem Untergang.

Zugleich wird in dieser Argumentations- und Diskursstruktur durchaus hintergründig Kultur in Natur verwandelt. Alles entwickelt sich gleichsam natürlich, gleichsam ohne das wirkliche Zutun des Menschen: Alles musste so sein, wie es war und wurde. Alles entwickelte sich, wie es von Natur aus programmiert war.

Und mehr noch: Die Welt Amerikas wird in ein Reich der Natur (zurück-) verwandelt, während Europa im Zeichen einer erfolgreich vorrückenden Kultur steht, die alles niederwirft, was sich ihrem Lauf entgegenstellt. Einem Reich der Zeichen steht ein Reich des Wissens, der Deutung dieser Zeichen, gegenüber. Nichts hiervon bleibt ungedeutet: Das Wissen der Europäer bemächtigt sich aller Dinge der Welt und macht sie sich untertan.

Aus dieser (europäischen) Kultur aber wird die Neue Welt folgenreich exkludiert. Wir haben es hier mit jener (gezielten) Konfusion von Biologie und Kultur zu tun, die Claude Lévi-Strauss in seiner Auseinandersetzung mit 'Rasse' und 'Geschichte' so stark betont hatte:

55 Ebda., Bd. I, S. a2v f.

56 Vgl. hierzu Church, Henry Ward: Corneille de Pauw, and the controversy over his 'Recherches philosophiques sur les Américains'. In: PMLA (New York) LI, 1 (March 1936), S. $180 \mathrm{f}$; sowie Beyerhaus, Gisbert: Abbé de Pauw und Friedrich der Große, eine Abrechnung mit Voltaire. In: Historische Zeitschrift (München - Berlin) 134 (1926), S. 465-493; sowie Moureau, François: L’Amérique n'a aucun avenir, S. 68. 
Mais le péché originel de l'anthropologie consiste dans la confusion entre la notion purement biologique de race (à supposer, d'ailleurs, que, même sur ce terrain limité, cette notion puisse prétendre à l'objectivité ce que la génétique moderne conteste) et les productions sociologiques et psychologiques des cultures humaines. ${ }^{57}$

An spektakulärer Inszenierung und überscharfer, dramatischer Beleuchtung ist de Pauws Argumentationsweise, die sich in ihrem weiteren Verlauf als ein gutes Beispiel für ein Rassedenken und vielleicht mehr noch einen Rassismus avant la lettre begreifen ließe, kaum zu überbieten. Dabei bilden die Recherches philosophiques sur les Américains ein Werk aus Worten, das sich auf keinen empirisch untersuchten Gegenstand, sondern ausschließlich auf andere Werke und Worte, auf andere Texte und intertextuelle Netzwerke bezieht. De Pauw hat Amerika nie betreten: Seine Kenntnis ist eine reine Textkenntnis, ein im Stile der Aufklärung kritischer Umgang mit Texten, zu denen ganz wesentlich Beispiele aus dem weiten Bereich der Reiseliteratur zählen. Cornelius de Pauw ist ein überzeugter Daheimgebliebener, der in Xanten oder Potsdam seine weltweiten Studien betrieb und die Reisenden selbst höchstens als Informationsquellen schätzte. Das Verhältnis zwischen Reisen und Schreiben ist bei ihm klar geregelt und an die eigene Sesshaftigkeit in Europa gebunden.

So steht das Werk de Pauws ein für eine Textwissenschaft im schwachen Sinne. Die Methode des Cornelius de Pauw, so ließe sich mithin sagen, war rein textbasiert: Sie ist in diesem Sinne eine philologische Methode und ihr Verfasser in erster Linie ein Philologe, der mit Texten arbeitet und sich mit Texten auskennt. Mit guten Gründen könnte man daher die Recherches philosophiques als eine nicht enden wollende Reise durch die Welt der Texte charakterisieren. Aber nicht durch die Welt einer außersprachlichen Wirklichkeit.

Ohne jegliche empirische Basis und ohne direkte Kenntnis der von ihm beschriebenen, diskutierten und bewerteten Gegenstände konsultierte de Pauw ebenso historische Chroniken wie zeitgenössische Reiseberichte, ebenso ihm zugängliche Bordbücher wie Manuskripte von Handelsreisenden, ebenso historiographische Werke des 16. Jahrhunderts wie philosophische Traktate des 18. Jahrhunderts. Seine Welt war die Welt der Archive und Bibliotheken, nicht die der Handelsstationen, der Karawansereien oder der fernen Hauptstädte anderer Zivilisationen.

Die Welt der Texte, in welcher sich de Pauw bewegt, wird durch seine Bewegungen eine Welt der Intertexte, welche miteinander in vielfältigen Beziehungen stehen. In diesen Bewegungen zwischen den Texten entsteht nicht nur

57 Lévi-Strauss, Claude: Race et histoire. Suivi de L'oeuvre de Claude Lévi-Strauss par Jean Pouillon. Paris: Denoël 1984, S. 10. 
eine gewisse Autonomie der von ihm durchquerten Textuniversen, sondern vielleicht mehr noch eine textuell erzeugte Autonomie und Eigen-Logik eines philosophe, der von einer erhöhten philosophischen Beobachterposition aus seine Urteile fällt und im Namen einer universalen Vernunft zu sprechen vorgibt. Denn die Herrschaft über die Texte generiert in ihm (und keineswegs nur in ihm) eine Art der Selbstermächtigung, eine Herrschaft über die Texte und die Menschen, die in diesen Texten vorkommen. Es geht um eine Beherrschung der Gegenstände im Sinne einer Beherrschung der Texte über diese Gegenstände. Denn Cornelius de Pauws 'Neue Welt' war eine Welt der Texte, auch wenn diese Welt keine Bibliothek (und schon gar keine 'Bibliothek von Babel') war. Ihr Schriftgelehrter, ihr Philologe aber war sich der Wege in der Welt seiner Bibliothek und damit der Wege in der Welt überhaupt ganz sicher.

Diese 'Neue Welt' wurde den rhetorischen Verfahren aufklärerischer Kritik ausgesetzt. Sie wurde so mmodelliert, wie sich de Pauw auch mit den alten Ägyptern oder auch mit China auseinandersetzte: durch seine 'kritischen' Studien von Texten ausgehend von einem Schema der Welt, das für ihn feststand und das er in den Windungen seiner Textwissenschaft Stück für Stück erhellte.

Dabei schenkte der sich zum damaligen Zeitpunkt in Potsdam und Berlin aufhaltende Holländer in aller Regel europäischen Stimmen weitaus mehr Glauben als Autoren, die - wie etwa Garcilaso de la Vega el Inca - amerikanischer Herkunft und für ihn schon deshalb disqualifiziert waren. Er setzte in französische Verfasser weit mehr Vertrauen als in spanische - und so schrieben sich seine Abstufungen auch innerhalb von Europa fort. Entscheidend für seinen kritischen Umgang mit den ihm zur Verfügung stehenden Texten aber war, dass es für de Pauw darauf ankam, einzelne Textbausteine zu finden, die für seine eigene, völlig unabhängig von empirischen Befunden getroffenen Einschätzungen amerikanischer Gegenstände nützlich und diskursstützend erschienen. Diese bildeten dann für ihn das Grundmuster seiner Argumentationen und damit eine Welt der Intertextualität, die für seine europäischen Zeitgenossen im 18. Jahrhundert durchaus überzeugend schien.

Folglich waren die von ihm hergestellten intertextuellen Relationen stets Beziehungen jener Macht, zitieren oder weglassen, affirmieren oder negieren, verlebendigen oder totschweigen zu können. Eine Herrschaft über Texte als eine Herrschaft über die Welt - und welche epistemische Bedeutung war schon dieser außersprachlichen Welt, der Augenzeugenschaft, der empirischen Erfahrung zuzubilligen? Lesen und Schreiben, textuelles Inkludieren und Exkludieren sind die grundlegenden Handlungen, die im Mittelpunkt des de Pauwschen Textuniversums stehen. An ihnen richtet sich alles aus.

Die Normen für die Beurteilung dessen, was in dieser Welt der Texte als glaubwürdig gilt oder als lügnerisch ausgeschlossen werden muss, können allein von 
einem aufgeklärten Europa, insbesondere auch von Berlin und Potsdam aus, definiert werden. Die Amerikaner sind Objekte, nicht aber Subjekte eines niemals auf Wechselseitigkeit beruhenden Diskurses, der ihnen mit Macht das Wort entzieht und zugleich an ihrer Stelle spricht. Sie verfügen über keine Stimme, haben keinen Diskurs: Sie sind Objekte dieser Textualität.

Cornelius de Pauws Bild des (indigenen) Amerikaners hat dabei nichts zu tun mit jenem des bon sauvage Rousseau'scher Prägung. Wir haben es vielmehr mit einem (im Sinne von Lévi-Strauss verstandenen) anthropologischen Entwurf zu tun, der diametral all dem entgegensteht, wofür vor de Pauw etwa ein Jean-Jacques Rousseau mit seinem Discours sur l'origine et les fondements de l'inégalité parmi les hommes ${ }^{58}$ oder nach de Pauw ein Bernardin de Saint-Pierre mit Paul et Virginie ${ }^{59}$ einzustehen versuchten. Werfen wir hier einen kurzen Blick auf eine Zusammenfassung von Charakterzügen, wie sie für 'den' Indianer de Pauws - und die Verwendung des Singulars ist wie auch in anderen Breichen seiner Recherches aufschlussreich - typisch sind und stereotyp wiederholt werden. Nachfolgend also eine für sein Schreiben typische Passage:

Il n'est proprement ni vertueux, ni méchant: quel motif auroit-il de l'être? La timidité de son ame, la foiblesse de son esprit, la nécessité de se procurer sa subsistance au sein de la disette, l'empire de sa superstition, \& les influences du climat l'égarent, \& l'égarent très-loin; mais il ne s'en aperçoit pas. Son bonheur est de ne pas penser, de rester dans une inaction parfaite, de dormir beaucoup, de ne se soucier de rien, quand sa faim est apaisée, \& de ne se soucier que des moyens de trouver la nourriture, quand l'appétit le tourmente. Il ne construiroit pas de cabane, si le froid \& l'inclémence de l'air ne l'y forçoient: il ne sortiroit pas de la cabane, s’il n'en étoit chassé par le besoin: sa raison ne vieillit pas: il reste enfant jusqu'à la mort, ne prévoit rien, ne perfectionne rien, \& laisse la nature dégénérer à ses yeux, sous ses mains, sans jamais l'encourager \& sans la tirer de son assoupissement. Fonbciérement paresseux par naturel, il est vindicatif par foiblesse, \& atroce dans sa vengeance, parce qu'il est lui-même insensible: n'ayant rien à perdre que la vie, il regarde tous ses ennemis comme ses meurtriers. ${ }^{60}$

Wir stoßen hier auf das exakte Gegenbild zu Antoine-Joseph Pernetys Bild des bon sauvage, den er selbst in den amerikanischen Tropen gesehen zu haben vorgab. Der Reduktion der unterschiedlichsten amerikanischen Kulturen auf

58 Vgl. Rousseau, Jean-Jacques: Discours sur l'origine et les fondements de l'inégalité parmi les hommes. In (ders.): Oeuvres complètes. Bd. III. Edition publiée sous la direction de Bernard Gagnebin et Marcel Raymond avec, pour ce volume, la collaboration de François Bouchardy, Jean-Daniel Candaux, Robert Derathé, Jean Fabre, Jean Starobinski et Sven Stelling-Michaud. Paris: Gallimard 1975.

59 Bernardin de Saint-Pierre, Jacques-Henri: Paul et Virginie. Paris: Editions Garnier Frères 1964.

60 Pauw, Cornelius de: Recherches philosophiques sur les Américains, Bd. 1, S. 123. 
das statische Bild ‘des' Indianers entspricht bei Cornelius de Pauw die Reduzierung dieses Menschen auf eine quasi tierische Existenz, die von keinerlei Entwicklung, keinerlei Dynamik und keinerlei Perfektibilität gekennzeichnet ist. Im Grunde verfügt dieser Mensch bestenfalls rudimentär über die Attribute des Menschseins in einem allgemeinen Sinne der civilisation des 18. Jahrhunderts. Daher wird es de Pauw auch nicht schwerfallen, eben diesen Menschen das Menschsein abzusprechen, sobald dies sein Diskurs notwendig macht.

Wir gelangen an dieser Stelle auch erneut an ein Grundverfahren des Cornelius de Pauw: Kultur wird in Natur umkodiert und damit zugleich zum Verschwinden gebracht. Damit wird 'der' Indianer, in einer unüberwindlichen Unmündigkeit gefangen, de facto aus der Geschichte der Menschheit ausgeschlossen, zu der er nichts beizutragen scheint, ja mehr noch: Er wird aus dem Menschengeschlecht ausgebürgert, eine Exklusion, die im zweiten, 1769 ebenfalls in Berlin erschienenen Band - wie wir in einem vorangehenden Zitat bereits gesehen haben - noch radikaler ins Werk gesetzt wird. 'Dem' Indianer wird keinerlei menschheitsgeschichtliche Bewegungsmöglichkeit zugesprochen: Im Denken von Cornelius de Pauw bleibt ihm die Zukunft definitiv verschlossen. Er ist ein Tier, ein Objekt, etwas, an dem Handlungen von Europäern, von wahren Menschen, vollzogen werden.

Doch selbst wenn wir die Indianer in die Menschheit einbezögen: Die somit letztlich zweigeteilte Menschheit ist ebenso unauflöslich aneinander gekettet wie die zweigeteilte Welt auf Gedeih und Verderb aufeinander bezogen und miteinander verwoben ist. Cornelius de Pauw entfaltet das Denken einer Globalität, die unaufhebbar von einer fundamentalen Gegensätzlichkeit geprägt ist und diese Opposition - auf der wissenschaftlichen Grundlage der Buffon'schen Histoire naturelle - in Begriffe vollständiger europäischer Superiorität und geradezu animalischer amerikanischer Inferiorität übersetzt. Kaum einer hat die Welt je schärfer aufgeteilt. Und kaum einer vermag, mit Hilfe dieser philologischen Rhetorik die Herrschaft der einen über die andere Welt mit den Mitteln einer europäischen Vernunft rhetorisch raffinierter zu begründen als der aus Amsterdam stammende Cornelius de Pauw.

Dabei wird die absolut gesetzte europäische Überlegenheit mit Blick auf die erste Phase beschleunigter Globalisierung auf der moralischen Ebene durchaus einer fundamentalen Kritik unterzogen. Denn das alte Amerika, „l'ancienne Amérique“, das noch die Zeitgenossen der Conquista kannten, bestehe längst nicht mehr, sei es doch „entièrement bouleversé par la cruauté, l'avarice, l'insaciabilité des Européens“. ${ }^{61}$ De Pauw baut Kritik ein, freilich eine vorwiegend

61 Pauw, Cornelius de: Recherches philosophiques sur les Américains, Bd. 1, S. a4r. 
rhetorische Kritik. Aus den spanischen Konquistadoren sind in diesem Zitat mit Bedacht Europäer geworden, und die von ihnen ausgehende zerstörerische Gewalt öffnet sich bei de Pauw sogleich auf mögliche Katastrophen von planetarischem Ausmaß.

Dies hat Folgen für die Argumentation des in Amsterdam Geborenen. Nicht nur die Weltgeschichte zeichnet sich im globalen Denken de Pauws ab, sondern auch der Weltenbrand. Hatte nicht die Syphilis, die der Abbé vielfach erwähnt und die wir heute als Leitepidemie der ersten Globalisierungsphase beschreiben können, auf eindrucksvolle Weise demonstriert, dass eine Krankheit sich dank weltweiter Schiffsverbindungen rasch zwischen Amerika, Europa und Afrika verbreiten konnte? Und war nicht anhand der Syphilis deutlich geworden, dass alle Bewohner der Erde in einem weltumspannenden Kalamitätenzusammenhang befindlich sind?

Vor allem aber im Zeichen der zweiten, zeitgenössischen Phase beschleunigter Globalisierung wird für de Pauw eine selbstverschuldete Auslöschung des gesamten Menschengeschlechts vorstellbar, eine „extinction totale“, ${ }^{62}$ die keiner Naturkatastrophe, sondern im Wesentlichen menschlichem Handeln geschuldet wäre. De Pauw führt gleichsam Vorstellungen der Apokalypse in die zeitgenössischen Szenarien der Weltentwicklung ein. Vor dem Hintergrund der kolonialen Expansion des 15. und 16. Jahrhunderts warnt der Verfasser der Recherches vor den Konsequenzen jener zweiten, sich von Europa aus über den Globus ausbreitenden Expansionswelle, als deren kritischen Zeitgenossen und Zuschauer sich der holländische Kleriker gerne selbst portraitiert. So ist seine 'Bestandsaufnahme' Amerikas mit apokalyptischen Zukunftsprognosen durchsetzt, die den Zeitgenossen wahre Schauer über den Rücken jagen mussten.

Wie sahen diese Visionen apokalyptischer Reiter bei ihm aus? In einer Zeit, in der wir selbst im Zeichen der Klimakatastrophe in weltumspannende apokalyptische Szenarien eingespannt sind, mag diese Frage vielleicht erlaubt sein. Nun, Europa stehe im Begriff, unter der Anleitung von „Politiques“ und unter dem Beifall mancher „Philosophes“ die „Terres Australes“ machtpolitisch und wissenschaftlich in Besitz $\mathrm{zu}$ nehmen, ohne $\mathrm{zu}$ bedenken, wieviel Unglück man notwendig über die dort lebenden Völker bringen werde. ${ }^{63}$ Damit eröffnet sich eine andere Seite der Kritik in Cornelius de Pauws Recherches philosophiques sur les Américains, eine Seite freilich, die bislang weder von de Pauws Zeitgenossen noch von

62 Ebda., Bd. I, S. a3r.

63 Ebda. 
der aktuellen Forschung berücksichtigt worden ist. ${ }^{64}$ Denn sie hat etwas Substanzielles mit dem aufklärerischen Projekt der Moderne und mit dem Zivilisationsvorhaben zu tun, für das Europa gerade im Siècle des Lumières stand.

Wichtig ist es, einen innerhalb des Denkens der europäischen Aufklärung überaus originellen Punkt zu beleuchten. Denn de Pauws Kritik trifft und betrifft keineswegs nur die europäische Expansionspolitik im militärischen und wirtschaftlichen Sinne, sondern auch und gerade die in Europa ansässigen Wissenschaften und deren jeweilige Eigeninteressen. Es geht also um Politiken des Wissens und der Wissenserzeugung. De Pauw spitzt diese Frage zu: Für die Aufklärung einiger strittiger geographischer Fragen oder die überprüfbare Durchführung von Temperaturmessungen sei man jederzeit und ohne größeres Zögern bereit, „la destruction d'une partie du globe“ in Kauf zu nehmen. ${ }^{65}$ Dieser Vorwurf des angriffslustigen Abbé ist ebenso berechtigt wie unerhört. Und unterliegt er nicht bis heute in allen Zentren abendländischer Wissenschaft einer offensichtlichen Tabuisierung?

Deutlich erscheint hier die Wissenschaft Europas in ihrer verselbständigten Eigenlogik als Impulsgeberin und Mittel europäischer Expansionspolitik zugleich. Sie ist alles andere als eine harmlose Beobachterin des europäischen Expansionsprozesses, die nur getreulich ihre Messungen vornimmt und ihre Daten aufzeichnet. Die Wissenschaft wird als Teil (des Problems) der europäischen Expansion selbst erkannt: Und dieser Gedanke ist hochinnovativ.

Cornelius de Pauw bleibt bei dieser Kritik nicht stehen, sondern sinnt auf Abhilfe. Einer derartigen, sich zeitgenössisch unstrittig beschleunigenden Entwicklung gelte es entgegenzutreten: „Mettons des bornes à la fureur de tout envahir, pour tout connoître. “66 Wissen ist hier nicht nur Macht, sondern trägt - auch und gerade im Erkenntniswillen europäischer Wissenschaft - den Keim und die Macht von Zerstörung und Selbstzerstörung in sich. Hier zeichnet sich zumindest in nuce eine Dialektik der Aufklärung ab, die über die von Horkheimer und Adorno gefassten Gedanken hinausgeht, aber zugleich mit ihnen in der Stoßrichtung übereinstimmt. Die Wissenschaft, so könnten und müssen wir folgern, ist keine unbeteiligte, quasi 'neutrale' und objektive Analytikerin der Expansion, sie ist ein wesentlicher Bestandteil dieser zerstörerischen Bewegung selbst, die

64 Vgl. Cañizares-Esguerra, Jorge: How to Write the History of the New World. Histories, Epistemologies, and Identities, in the Eighteenth-Century Atlantic World. Stanford: Stanford University Press 2001, S. 27; erste Ansätze in Ette, Ottmar: „Not just brought about by chance“: reflections on globalisation in Cornelius de Pauw and Alexander von Humboldt. In: Studies in Travel Writing (Nottingham) XV, 1 (February 2011), S. 3-25.

65 Pauw, Cornelius de: Recherches philosophiques sur les Américains, Bd. I, S. a4v. 66 Ebda. 
folglich - so ließe sich hinzufügen - das friedvolle Zusammenleben auf unserem Planeten beeinträchtigt und gefährdet. Das ist ein ernst zu nehmender, veritabler Angriff gegen ein europäisches Verständnis von Wissenschaft.

Der scharfzüngige holländische Kleriker, der sich zweimal - zunächst 1767 und 1768 sowie 1775 und 1776 - am Hofe Friedrichs des Großen in Berlin und Potsdam der königlichen Gunst erfreute und von Antonello Gerbi später auch mit Blick auf seine nachfolgenden langen Jahre in Xanten als „abbate prussiano“67 tituliert wurde, erkannte die zerstörerische und selbstzerstörerische Logik einer Entwicklung, in deren Kontext das, was in Europa vor sich geht, unmittelbare Folgen und Auswirkungen auf die ganze Welt, auf die gesamte Menschheit zeitigen könne. Die zerstörerische Kraft europäischer Wissenschaft, eines Wissens um jeden Preis, solle und müsse eingedämmt werden. Aber wie?

De Pauws Warnung war an Deutlichkeit kaum zu überbieten. Längst seien die politischen und wirtschaftlichen Interessengegensätze zwischen den Europäern gerade in den Kolonialgebieten so ausgeprägt, dass es nur eines Funkens bedürfe, um die ganze Welt von einem bis zum anderen Ende in Brand zu setzen:

[...] une étincelle de discorde, pour quelques arpents de terre au Canada, enflamme et embrase l'Europe; \& quand l'Europe est en guerre, tout l'Univers y est: tous les points du globe sont successivement ébranlés comme par une puissance électrique: on a agrandi la scene des massacres et du carnage depuis Canton jusqu'à Archangel; depuis BuénosAires jusqu'à Quebec. Le commerce des Européens ayant intimement lié les différentes parties du monde par la même chaîne, elles sont également entraînées dans les révolutions \& les vicissitudes de l'attaque \& de la défense, sans que l'Asie puisse être neutre, lorsque quelques marchands ont des querelles en Amérique, pour des peaux de Castor, ou du bois de Campèche. ${ }^{68}$

Dieselbe Kette („une même chaîne“) verbindet die Welt und bindet sie als Fessel auf Gedeih und Verderb zusammen. Globalität ist das Zeichen jener Philosophie, um die sich de Pauw bemüht, deren Dimensionen er zu erkunden sucht. Ein Außerhalb des Planeten gibt es für ihn jedenfalls nicht.

Konflikte im Welthandel, dies hatte sich im 18. Jahrhundert bereits gezeigt, konnten unversehens zu militärischen Konfrontationen eskalieren, die man mit Fug und Recht als Weltkriege bezeichnen darf. Lokal oder regional scheinbar begrenzte Anlässe, so de Pauw, reichten folglich aus, um europäische Auseinandersetzungen global auszufechten und den Krieg in die nur auf den ersten Blick entferntesten und entlegensten Gebiete beider Hemisphären zu tragen. Die Bewohner der außereuropäischen Welt erscheinen hierbei niemals als

67 Gerbi, Antonello: La Disputa del Nuovo Mondo. Storia di una Polemica: 1750-1900, S. 117. 68 Pauw, Cornelius de: Recherches philosophiques sur les Américains, Bd. I, S. 90. 
Subjekte, sondern bestenfalls als Gehandelte, als Objekte fremden Tuns in einer von Europa aus gelenkten Weltpolitik, die längst selbst ihren wichtigsten 'Machern' und Akteuren zu entgleiten droht. Denn der Weltenbrand ist die Konsequenz von Handlungsweisen, die den wichtigsten Akteuren in Europa immer mehr aus den Händen geglitten sind.

Bei Cornelius de Pauw zeichnet sich ein höchst aufschlussreiches Verständnis abendländischer Wissenschaft ab. Denn diese ist niemals nur bloßes Instrument einer anderen, insbesondere einer politischen Macht: Sie ist stets auch eine Macht für sich und verfolgt eigene Interessen, die durchzusetzen sie in der Lage ist. Die Kritik des Klerikers an der europäischen Expansion wie an der europäischen Wissenschaft schloss freilich die Kritik an der eigenen Wissenschaft, an der de Pauw'schen Wissenschaftspraxis also, nicht mit ein. Wohl aus guten Gründen.

Denn daran, dass de Pauw für seinen eigenen Ansatz im Allgemeinen und für seine philosophischen Recherches im Besonderen den Status des Wissenschaftlichen einforderte, kann keinerlei Zweifel bestehen. Er begriff sich als ein typisches Kind der Aufklärung, das Licht ins zuvor Dunkle bringen wollte. So hielt er in der nach der Veröffentlichung seines zweiten Bandes ausbrechenden Berliner Debatte um die Neue Welt seinem ärgsten Widersacher im dritten, bereits 1770 erschienenen Band seiner Recherches philosophiques sur les Américains entgegen, dieser habe weder die Wissenschaftlichkeit seines Ansatzes erkannt noch auf der Höhe seiner Wissenschaft argumentiert. ${ }^{69}$ Die sich gegen einen bloß 'parlierenden', ohne Methode und System vor sich hinschreibenden Gegner wendende Replik de Pauws fiel daher vernichtend aus:

A l'entendre parler, il semble qu'il lui suffisoit de prendre la plume pour composer une réfutation dans les formes [...]. Aussi ne donne-t-il aucune observation sur l'Histoire Naturelle: il a mieux aimé employer la morale, des compilations extraites du compilateur Gueudeville, \& enfin des raisonnements à perte de vue.

Quand on attaque un livre écrit sur une science, il faut se servir d'arguments tirés de cette science, $\&$ non d'une autre. ${ }^{70}$

Aufschlussreich an dieser Argumentationsweise ist, dass sich de Pauw hier nicht allein auf die im Zeichen Buffons stehende Wissenschaft der Histoire

69 Diese neue Ausgabe von 1770 wurde in den Folgejahren mehrfach auch an anderen Druckorten wieder aufgelegt.

70 Pauw, Cornelius de: Recherches philosophiques sur les Américains ou Mémoires intéressants pour servir à l'Histoire de l'Espéce Humaine. Par Mr. de P***. Avec une Dissertation sur l'Amérique \& les Américains, par Dom Pernety. Et la Défense de l'Auteur des Recherches contre cette Dissertation. Bd. III. Berlin 1770, S. 11. 
naturelle, sondern auch auf seine eigene philologische Vorgehensweise beruft, die sich - anders als sein Widersacher - niemals unkritisch irgendwelcher Kompilationen bedient habe. Diese Wissenschaft einer philologisch-kritischen Arbeit am Text jedoch unterwirft der holländische philosophe keinerlei eigener Kritik mehr: Seine Kritik, seine Wissenschaft wird sozusagen nicht selbstreflexiv. Er überprüft mithin auch nicht, welche weltweiten Folgen seine eigenen wissenschaftlichen Interessen - parallel zu den Interessen jener Wissenschaften, die sich auf Gegenstände der Natur (wie Temperatur und Territorium) richten - zeitigen könnten. War seine Wissenschaft aber dann überhaupt noch eine Wissenschaft? Wie schwerwiegend diese Konsequenzen sein konnten, sollte der weitere Fortgang der von den Recherches philosophiques ausgelösten Debatte in aller Deutlichkeit vor Augen führen.

Halten wir zunächst einmal fest: Die von Berlin aus weltweit anhebende Debatte zeigt mit aller Deutlichkeit, dass Aufklärung kein rein europäisches Phänomen ist. Dabei demonstrierte diese auf den ersten Blick lokale, periphere Debatte zugleich, dass es unzureichend ist, wenn man sich alleine auf die europäische Aufklärung konzentriert: Denn nur von Europa aus ist auch das Phänomen der Aufklärung nicht adäquat zu durchdringen und zu begreifen. Längst hatte die République des Lettres einen transarealen Zuschnitt: Was in Berlin diskutiert wurde, konnte und musste auch die Gemüter in Mexico-Stadt, in Philadelphia oder in Lima erhitzen. Und zu schriftlichen Reaktionen führen. Vergessen wir also nicht, dass die Aufklärung im Zeichen der zweiten Phase beschleunigter Globalisierung ein tendenziell weltweites, weltumspannendes Phänomen darstellt, das auch in seinen lokalen Entwicklungen translokale und damit letztlich transareale Dimensionen beinhaltet und entfaltet. Dies veranschaulicht sehr genau die Berliner Debatte um die Neue Welt.

Die ersten Runden dieses von Berlin ausgehenden und in Berlin geführten Disputs gingen ohne Frage an Cornelius de Pauw. Denn dieser hatte nur auf die Chance eines Gegenangriffs, der im Wesentlichen seine Abwertung der Amerikaner in ein idealisierendes Gegen-Bild alles Amerikanischen umzukehren suchte, gewartet und druckte großzügig Pernetys Attacke in seiner bereits erwähnten und noch 1770 wiederum in Berlin veröffentlichten dreibändigen Neuausgabe seiner Recherches philosophiques sur les Américains ab. Ihr fügte er eine vehemente und doch wohlkalkulierte Verteidigungsschrift seiner eigenen, vielfach weiter zugespitzten Thesen bei. Pernetys über weite Strecken inverse Darstellung der Thesen seines Kontrahenten konnte von ihm leicht diskursiv vereinnahmt werden.

Es ist sicherlich nicht ganz fair, spricht man Antoine-Joseph Pernety jegliche Berechtigung $\mathrm{zu}$ einem philosophisch ernst $\mathrm{zu}$ nehmenden Diskurs ab. Und gewiss darf man bei Pernety „elements of a modern ethnological 
attitude“71 erkennen, zu denen man sicherlich „the critique of Eurocentrism; the importance of gathering data in the field and verifying sources; the recognition of the cultural diversity of the Other"72 rechnen kann. Doch blieb seine Argumentationsweise - wie wir bereits sahen - allzu sehr an die von de Pauw ins Feld geführten Thesen gebunden, die der Bibliothekar Friedrichs II. oftmals nur mit anderen Vorzeichen versah, ohne ihnen eine neue argumentative (und wissenschaftlich begründbare) Fundierung zu geben.

Damit wurde de Pauw zum Mann der Stunde. Ungeachtet aller weiteren Publikationen von Pernety, die freilich kaum noch Wesentliches zur Debatte beitrugen, ${ }^{73}$ sollte sein Werk noch für lange Jahre im Zentrum der von ihm ausgelösten hitzigen und weltweit erbittert geführten Debatten um Amerika und die Amerikaner stehen, bevor es dann in ein im Grunde bis in die jüngste Vergangenheit andauerndes Vergessen fiel. Nicht umsonst aber erklärte die französische Nationalversammlung in einem Dekret vom 26. August 1792 Cornelius de Pauw gemeinsam mit anderen um die Freiheit der Völker verdienten Persönlichkeiten, zu denen auch George Washington zählte, zum citoyen français. ${ }^{74}$ In Europa hatte der holländische Abbé seine Partie gewonnen. Und noch Napoléon ließ ihm zu Ehren in Xanten einen Obelisken errichten, der an die Verdienste des Philosophen erinnern sollte.

Grundsätzliche und weit über den Horizont Pernetys hinausgehende Einund Widersprüche gegen Cornelius de Pauws Thesen ließen noch im 18. Jahrhundert nicht lange auf sich warten. So veröffentlichte etwa der aus Neuspanien ausgewiesene Jesuit Francisco Javier Clavijero in seinem italienischen Exil 1780 eine umfangreiche Geschichte des alten Mexico, ${ }^{75}$ in welcher er eindrucksvoll die Diversität der indigenen Kulturen Amerikas auf dem Gebiet des heutigen Mexico aufzeigte. Dabei gelang es diesem neuspanischen Autor nicht nur, den Thesen von de Pauw, aber auch von Raynal oder Robertson dadurch entgegenzutreten, dass in breiter Vielfalt amerikanische Quellen einschließlich der Bilderhandschriften und anderer indigener Dokumente miteinbezogen wurden;

71 Mannucci, Erica Joy: The savage and the civilised: observations on a dispute between an enlightened writer and an illuminist. In: Studies on Voltaire and the Eighteenth Century (Oxford) 303 (1992), S. 384.

72 Ebda.

73 Vgl. u. a. Pernety, Antoine-Joseph: Examen des Recherches philosophiques sur les Américains et de la Défense de cet ouvrage. 2 Bde. Berlin: G.J. Decker, Imprimeur du Roi 1771.

74 Vgl. hierzu Moureau, François: L'Amérique n’a aucun avenir, S. 68.

75 Vgl. Clavijero, Francisco Javier: Storia Antica del Messico. 4 Bde. Cesena: Gregorio Biasani 1780. 
er trieb vielmehr eine Konstruktion der amerikanischen Vergangenheit voran, die als - im besten Sinne - Findung und Erfindung einer anderen Herkunft auch eine andere Zukunft für seine amerikanische Heimat ermöglichen sollte. Die Perspektive der Berliner Debatte begann sich neuweltlich zu drehen.

Die präkolumbischen Kulturen erschienen nun nicht länger als vernachlässigbare Randerscheinungen der Menschheitsgeschichte, sondern stellten sich selbstbewusst als amerikanische Antike einer europäischen Antike gegenüber. So wurden die indigenen amerikanischen Kulturen wieder in Bewegung gesetzt und eröffneten neue Perspektiven und Einsichten in eine andere, von Amerika aus zu gestaltende Zukunft. Hier werden die hochgradig produktiven Akzente der Berliner Debatte, die sich längst von Berlin entfernt hatte, deutlich.

Doch entwickelte sich die vielleicht entscheidende Widerstandsfront gegen die Vorstellungen de Pauws ausgehend von Berlin selbst. Denn es wäre ein Leichtes zu belegen, dass den in der Berliner Debatte vorgetragenen Positionen bereits jene Schriften, die der philosophe voyageur Alexander von Humboldt wenige Jahre nach der Rückkehr von seiner Reise in die amerikanischen Tropen (1799-1804) veröffentlichte, den wissenschaftlichen Todesstoß versetzten.

So notierte der weitgereiste Gelehrte in seiner auf Paris im April 1813 datierten Einleitung in seine Vues des Cordillères et Monumens des Peuples Indigènes de l'Amérique mit großer Klarheit:

L'ardeur avec laquelle on s'étoit livré à des recherches sur l'Amérique, diminua dès le commencement du dix-septième siècle; les colonies espagnoles, qui enferment les seules régions jadis habitées par des peuples civilisés, restèrent fermées aux nations étrangères; et récemment, lorsque l'abbé Clavigero publia en Italie son Histoire ancienne du Mexique, on regarda comme très-douteux des faits attestés par une foule de témoins oculaires souvent ennemis les uns des autres. Des écrivains célèbres, plus frappés des contrastes que de l'harmonie de la nature, s'étoient plu à dépeindre l'Amérique entière comme un pays marécageux, contraire à la multiplication des animaux, et nouvellement habité par des hordes aussi peu civilisées que les habitans de la mer du Sud. Dans les recherches historiques sur les Américains, un scepticisme absolu avoit été substitué à une saine critique. On confondoit les descriptions déclamatoires de Solis et de quelques autres écrivains qui n'avoient pas quitté l'Europe, avec les relations simples et vraies des premiers voyageurs; il paroissoit du devoir d'un philosophe de nier tout ce qui avoit été observé par des missionnaires.

Depuis la fin du dernier siècle, une révolution heureuse s'est opérée dans la manière d'envisager la civilisation des peuples et les causes qui en arrêtent ou favorisent les progrès. Nous avons appris à connoître des nations dont les moeurs, les institutions et les arts diffèrent presque autant de ceux des Grecs et des Romains, que les formes primitives d'animaux détruits diffèrent de celles des espèces qui sont l'object de l'histoire naturelle descriptive. La société de Calcutta a répandu une vive lumière sur l'histoire des peuples de l'Asie. Les monumens de l'Egypte, décrits de nos jours avec une 
admirable exactitude, ont été comparés aux monumens des pays les plus éloignés, et mes recherches sur les peuples indigènes de l'Amérique paroissent à une époque où l'on ne regarde pas comme indigne d'attention tout ce qui s'éloigne du style dont les Grecs nous ont laissé d'inimitables modèles. ${ }^{76}$

Diese Passage markiert einen freilich vorläufigen Schlusspunkt in jener Berliner Debatte, in die Antoine-Joseph Pernety, 'Le Philosophe la Douceur' alias Zaccaria de Pazzi de Bonneville, ${ }^{77}$ Giovanni Rinaldo Carli, Fray Servando Teresa de Mier y Guerra, Delisle de Sales, Francisco Javier Clavijero, George Washington, Drouin de Bercy und viele andere auf beiden Seiten des Atlantik eingegriffen hatten. Längst war die Berliner Debatte zu einer weltweiten Auseinandersetzung um das Verhältnis zwischen Alter und Neuer Welt, zwischen europäischen und amerikanischen Vorurteilen, aber auch zwischen europäischen und amerikanischen philosophes und Wissenschaftlern geworden. Unsere leider noch immer aktuellen Debatten und Dispute um einen mehr oder minder diskreten Eurozentrismus schreiben sich in diese lange Zeit verschüttete Traditionslinie ein.

Mit unverkennbaren Anspielungen auf die Werke de Pauws und Raynals signalisierte Alexander von Humboldt nicht nur die Entstehung eines neuen Diskurses über die Neue Welt, in dessen Geschichte der Name Clavijeros nicht fehlen durfte, sondern machte auch darauf aufmerksam, daß mit Blick auf 'Westindien' wie auf 'Ostindien' neue, empirische Grundlagen der Zirkulation von Wissen über die gesamte außereuropäische Welt entstanden waren. Eine neue Epoche, eine Epoche nach der 'glücklichen Revolution', war angebrochen. Das Bewusstsein, sein eigenes Werk in einer veritablen Epochenschwelle vorzulegen und zu dieser etwas Substanzielles beizutragen, hätte bei Alexander von Humboldt kaum ausgeprägter sein können. Humboldt wusste, dass seine amerikanistischen Schriften eine produktive und neue Horizonte eröffnende Antwort auf diese Phase beschleunigter Globalisierung waren.

Die in der angeführten Passage in dichter Folge aufgeführten Aspekte des Neubeginns wurden - wie wir sahen - in die heitere Semantik einer 'glücklichen Revolution' (die sich zutiefst von jener „étonnante révolution“ unterscheidet, von der de Pauw zu Beginn seines Werkes sprach) ${ }^{78}$ gerückt. Zug um Zug

76 Humboldt, Alexander von: Vues des Cordillères et Monumens des Peuples Indigènes de l'Amérique. Nanterre: Editions Erasme 1989, S. 96, S. II f.

77 Vgl. hierzu Zantop, Susanne: Colonial Fantasies. Conquest, Family, and Nation in Precolonial Germany, 1770-1870. Durham - London: Duke University Press 1997, S. 47.

78 Pauw, Cornelius de: Recherches philosophiques sur les Américains, ou Mémoires intéressants pour servir à l'Histoire de l'Espèce humaine, Bd. I, S. a2v. 
entkräfteten neue Dokumente in den Archiven und Bibliotheken der Neuen wie der Alten Welt, aber auch die Feldforschungen vieler amerikanischer wie europäischer Gelehrter und Reisender vor Ort, die unhaltbar gewordenen Thesen des niederländischen Philosophen, dessen Name in den Schriften Humboldts kaum mehr genannt zu werden brauchte. De Pauw schien erledigt. Sein Name geriet in Vergessenheit.

Die Berliner Debatte hatte zu einem weltweiten Disput geführt, der in seinem Ergebnis wesentlich zu der von Humboldt beobachteten und belegten wissenschaftlichen Revolution beigetragen hatte. Ohne die Erfahrung und das Erleben der zweiten Phase beschleunigter Globalisierung und ihrer Konsequenzen hätte sich diese Wissenschaftsrevolution nicht auf eine so durchschlagende Weise vollzogen. Dies bedeutete freilich keineswegs, dass einige der Grundgedanken von Cornelius de Pauw nicht doch überlebt und etwa in die Philosophie Hegels massivsten Eingang gefunden hätten. Aber dieses Weiterleben de Pauws braucht uns in dieser Vorlesung über Reiseliteratur nicht mehr ausführlich $\mathrm{zu}$ interessieren.

Doch sollte uns all dies nicht dazu verleiten, die Auswirkungen gerade auch der Thesen des Cornelius de Pauw zu unterschätzen. Denn dem Hauptwerk de Pauws kam zweifellos eine zwar diffuse, aber langanhaltende und nicht selten subkutane Wirkung zu, die sich keinesfalls an der expliziten Bezugnahme auf den Namen des Holländers festmachen lässt. Es wäre daher keinesfalls übertrieben, in Weiterentwicklung der Überlegungen Antonello Gerbis ${ }^{79}$ von einer diffusen Langzeitwirkung zu sprechen, die sich insbesondere auf die Philosophie, aber auch auf den Spannungsraum von Philologie und Rassismus beziehen ließe.

Denn das Inferioritätsdenken de Pauws blieb mit Blick auf die außereuropäische Welt nicht nur in Hegels berühmten Vorlesungen über die Philosophie der Weltgeschichte noch lange in Europa lebendig. Mit der Einsicht in das HistorischGewordensein der Berliner Debatte verbindet sich heute die Notwendigkeit, ihr historisches Gewordensein polylogisch und relational, kritisch und selbstkritisch an den Anforderungen unserer Phase beschleunigter Globalisierung zu überprüfen, um die konstruktive, kreative - und nicht die zerstörerische - Macht der Wissenschaften zu nutzen. In diesem Geiste wollen wir unsere Überlegungen zur Berliner Debatte um die Neue Welt beschließen.

79 Vgl. hierzu Gerbi, Antonello: La Disputa del Nuovo Mondo, S. 118 f. 
Dabei gilt es, in einer ganz grundlegenden Weise Aufklärung als ein weltumspannendes Epochenphänomen zu begreifen, wobei man allen Versuchungen widerstehen sollte, Vertreter der Aufklärung europäischer oder amerikanischer Herkunft schematisch einander entgegenzustellen und unter Missachtung der tatsächlichen Komplexität der Diskussionen, Diskurse und Debatten je nach eingenommenem Blickwinkel positiv oder negativ zu bewerten. Auch mit Blick auf diese Herausforderung ist die Berliner Debatte noch längst nicht zu einem Ende gekommen. Sie beinhaltet eine Reflexion über das Verhältnis zwischen Reisen und Schreiben, das uns auch in den nachfolgenden Kapiteln begleiten wird. 